\title{
Weinstein manifolds revisited
}

\author{
Yakov Eliashberg* \\ Department of Mathematics, Stanford University
}

\begin{abstract}
This is a very biased and incomplete survey of some basic notions, old and new results, as well as open problems concerning Weinstein symplectic manifolds.
\end{abstract}

\section{Weinstein manifolds, domains, cobordisms}

We begin with a notion of a Liouville domain. Let $(X, \omega)$ be a $2 n$-dimensional compact symplectic manifold with boundary equipped with an exact symplectic form $\omega$. A Liouville structure on $(X, \omega)$ is a choice of a primitive $\lambda, d \lambda=\omega$, called Liouville form such that $\left.\lambda\right|_{\partial X}$ is a contact form and the orientation of $\partial X$ by the form $\left.\lambda \wedge d \lambda^{n-1}\right|_{\partial X}$ coincides with its orientation as the boundary of symplectic manifold $(X, \omega)$. The vector field $Z$, that is $\omega$-dual to $\lambda$, i.e. $\iota(Z) \omega=\lambda$, is also called Liouville. It satisfies the condition $L_{Z} \omega=\omega$ which means that its flow is conformally symplectically expanding. The contact boundary condition is equivalent to the outward transversality of $Z$ to $\partial X$. A Liouville domain $X$ can always be completed to a Liouville manifold $\widehat{X}$ by attaching a cylindrical end:

$$
\widehat{X}:=X \cup(\partial X \times[0, \infty))
$$

and extending $\lambda$ to $\widehat{X}$ as equal to $e^{s}\left(\left.\lambda\right|_{\partial X}\right)$ on the attached end. We will be constantly going back and forth between these two tightly related notions of Liouville domains and Liouville manifolds.

Given a Liouville structure $\mathfrak{L}=(X, \omega, Z)$ we say that a Liouville structure $\mathfrak{L}^{\prime}=$ $\left(X^{\prime}, \omega, Z\right)$ is obtained by a radial deformation from $\mathfrak{L}$ if there exists a function $h$ :

*Partially supported by NSF grant DMS-1505910 
$\widehat{X} \rightarrow \mathbb{R}$ such that $X^{\prime} \subset \widehat{X}$ is the image of $X$ under the time 1 map $\psi: \widehat{X} \rightarrow \widehat{X}$ of the flow of the vector field $h Z$ on the completion $\widehat{X}$. The completions of the radially equivalent Liouville domains $\mathfrak{L}^{\prime}$ and $\mathfrak{L}$ are canonically isomorphic.

The space of Liouville structures for $(X, \omega)$ is convex, and hence any two Liouville structures are canonically homotopic. Given a homotopy of completed Liouville structures $\left(\widehat{X}, \omega_{t}, \lambda_{t}\right)$ there exists an isotopy $\phi_{t}: \widehat{X} \rightarrow \widehat{X}$ such that $\phi_{t}^{*} \omega_{t}=\omega_{0}$. Moreover, one can always arrange that $\phi_{t}^{*} \lambda_{t}=\lambda_{0}+d H_{t}$, see [11], Sections 11.1 and 11.2. In particular on completed Liouville manifolds it is always sufficient to consider homotopies fixing the symplectic form, and, moreover, changing the Liouville form by adding an exact form. Homotopic non-completed Liouville domains are symplectomorphic up to radial deformation.

Given a Liouville domain $\mathfrak{L}=(X, \omega, \lambda)$ consider a compact set

$$
\operatorname{Core}(\mathfrak{L})=\bigcap_{\mathrm{t}>0} \mathrm{Z}^{-\mathrm{t}}(\mathrm{X})
$$

the attractor of the negative flow of the Liouville vector field $Z$. We will call Core $(\mathfrak{L})$ the core, or the skeleton of the Liouville structure $\mathfrak{L}$. While Core $(\mathfrak{L})$ has obviously its $2 n$-dimensional Lebesgue measure equal to 0 , it still can be pretty large if no extra conditions are imposed on the Liouville structure. For instance, McDuff constructed in 31] a Liouville structure on $T^{*} S_{g} \backslash S_{g}$ for a closed surface $S_{g}$ of genus $g>1$, whose core has codimension 1 .

However, the situation changes if one requires existence of a Lyapunov function for the Liouville vector field $Z$. A Weinstein structure on a domain $X$ is a Liouville structure $\mathfrak{L}$ together with a function $\phi: X \rightarrow \mathbb{R}$ which is Lyapunov for the Liouville vector field $Z$, i.e.

(L1) $d \phi(Z)>c\|Z\|^{2}$ for a positive constant $c$ and some Riemann metric on $X$.

Note that condition (L1) implies that $\operatorname{Core}(\mathrm{X}, \lambda)$ is the union of $Z$-stable manifolds of critical points of $\phi$ (i.e. points converging to the critical locus in forward time). In [11] it was required in addition that $\phi$ is either Morse or generalized Morse (i.e. may have death-birth critical points). Under these assumptions it was shown in [1], see also [20, 15], that

(L2) the core is stratified by isotropic for $\lambda$, and hence for $\omega$ submanifolds.

F. Laudenbach proved, see [30], that if the flow of $Z$ is Morse-Smale (i.e. stable and unstable manifolds of critical points intersect transversely) and near critical points 


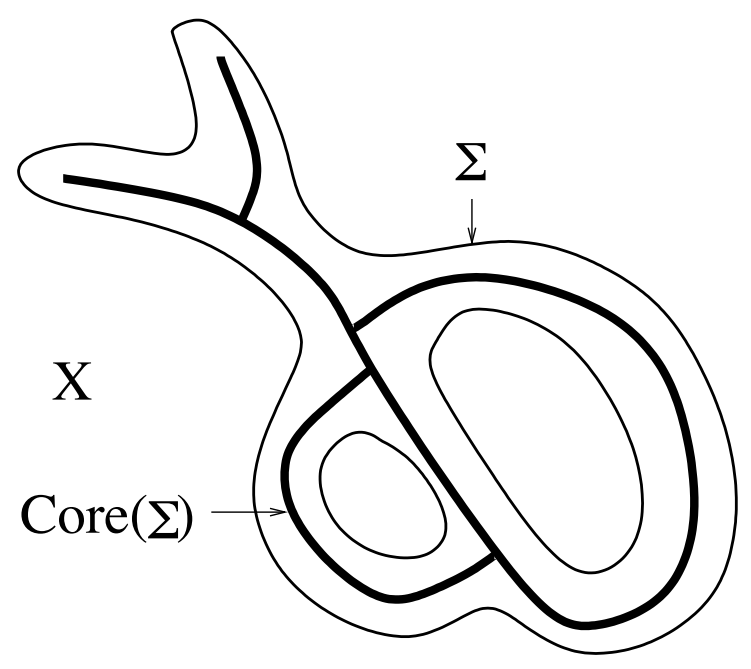

Fig. 1.1: Skeleton of a Weinstein domain

the vector field $Z$ is gradient with respect to an Euclidean metric, then the skeleton can be further Whitney substratified. It is likely that the Whitney condition also holds if near its zeroes the vector field $Z$ is gradient with respect to any Riemannian metric. However, as far as know, this was never verified in the literature. The Whitney condition need not hold if eigenvalues of the linearization of $Z$ at critical points have non-vanishing imaginary parts, as a spiraling phenomenon of trajectories may occur. 1

Condition (L2) holds for a much more general class of taming functions (e.g. when $\phi$ is Morse-Bott), and hence for the the purposes of this paper we will take the following working definition of a Weinstein structure, extending the class considered in [11]: $\mathfrak{W}=(X, \lambda, Z, \phi)$ is Weinstein if it satisfies conditions (L1) and (L2) with the Whitney condition and also condition

(L3) there exists a smooth family of Weinstein structures $\mathfrak{W}_{t}=\left(X, \lambda_{t}, \phi_{t}\right), t \geq 0$ such that $(\lambda, \phi)=\left(\lambda_{0}, \phi_{0}\right)$ and $\phi_{t}$ is Morse for $t>0$.

Problem 1.1. Which conditions (or maybe none?) on $\phi$ and $Z$ are needed to deduce (L2) and (L3) from (L1)?

A. Oancea suggested to me that a good sufficiently general condition on a Weinstein structure could be to require that near critical points it is generated by a $J$-convex

\footnotetext{
${ }^{1}$ I thank Francois Laudenbach for the discussion of the involved issues.
} 
function with respect to some (not necessarily integrable) almost complex structure $J$, see [11], Chapter 1, for the details.

Remark 1.2. Note that not every closed subset $C$ of a symplectic manifold which is stratified by isotropic strata may serve as the skeleton for an appropriately chosen Weinstein structure on a neighborhood of $C$ (compatible with the given ambient symplectic form). Examples of this kind exist already in $\mathbb{R}^{2}$. For instance, let

$$
C:=\{x=0, y \geq 0\} \cup\left\{x=y^{2}, y \geq 0\right\} \cup\{y=0, x \geq 0\} \cup\left\{y=x^{2}, x \geq 0\right\}
$$

be the union of 4 arcs emanating from the origin. Then there is no Liouville structure on a neighborhood $U \ni 0$ which has $C \cap U$ as a part of its skeleton. Indeed it is straightforward to check that for any 1-form $\lambda$ vanishing on $C \cap U$ one has $(d \lambda)_{0}=0$.

Problem 1.3. Find a necessary and sufficient condition on a compact subset $C$ of a symplectic manifold to serve as the skeleton of some

a) Liouville, or

b) Weinstein

structure on its neighborhood. In particular, is it true that a Whitney stratified subset $C$ which is the skeleton of a Liouville structure on its neighborhood also serves as the skeleton of a Weinstein structure?

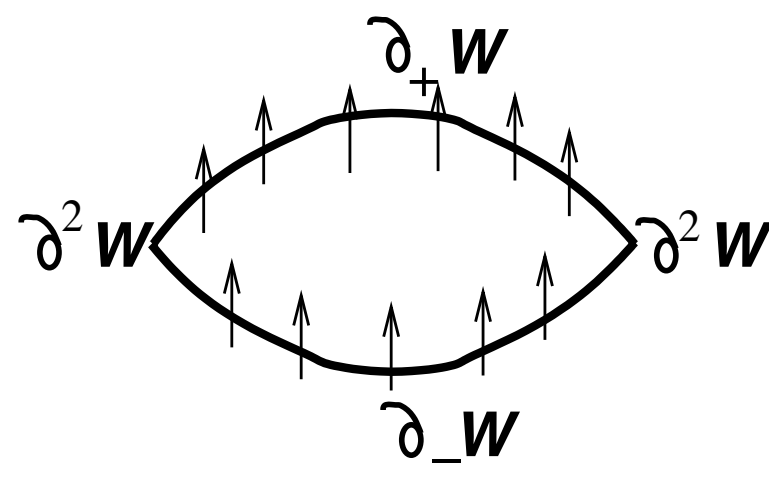

Fig. 1.2: Sutured Weinstein cobordism $W$ with corners.

It is also useful to consider a notion of a Weinstein cobordism. This is a cobordism $\left(W, \partial_{-} W=Y_{-}, \partial_{+} W=Y_{+}\right)$endowed with a Liouville form $\lambda$, whose Liouville vector 
field $Z$ is outward transverse to $\partial_{+} W$ and inward transverse to $\partial_{-} W$, and a Lyapunov (i.e. satisfying condition (L1)) function $\phi: W \rightarrow \mathbb{R}$ for the field $Z$. We also postulate (L3) and an analog of condition (L2) for the core of the Weinstein cobordism, which we define in that case as the stable manifold of the critical locus of $\phi$. We will also be considering Weinstein cobordisms between manifolds with boundary $\partial_{ \pm} W$. We will view these cobordisms as sutured manifolds with a corner along the suture, see Fig. 1.2. More precisely, we assume that the boundary $\partial W$ is presented as the union of two manifolds $\partial_{-} W$ and $\partial_{+} W$ with common boundary $\partial^{2} W=\partial_{+} W \cap \partial_{-} W$ along which it has a corner. The vector field $Z$ transversely enters $W$ through $\partial_{-} W$ and exits through $\partial_{+} W$, but of course, in this case the function $\phi$ cannot be chosen constant on $\partial_{-} W$ and $\partial_{+} W$.

While any two Weinstein structures on the same symplectic manifold are (canonically) homotopic as Liouville structures, the problem of existence of a Weinstein homotopy is widely open.

Problem 1.4. Let $\left(\widehat{X}, \lambda_{0}, \phi_{0}\right)$ and $\left(\widehat{X}, \lambda_{1}, \phi_{1}\right)$ be two completed Weinstein structures on the same symplectic manifold $(\widehat{X}, \omega)$. Are they homotopic as Weinstein structures?

In particular,

Problem 1.5. Let $\mathfrak{W}=(\widehat{X}, \omega, \lambda, \phi)$ be a completed Weinstein structure, and $f$ : $\widehat{X} \rightarrow \widehat{X}$ a symplectomorphism. Is the pull-back Weinstein structure $f^{*} \mathfrak{W}$ is Weinstein homotopic to $\mathfrak{W}$ ?

The Weinstein structure notion was introduced in [20] as a symplectic counterpart of the notion of Stein complex structure, and inspired by the work of A. Weinstein [45], see also [16, 15, 11].

I discussed the notions and problems considered in this paper with many people. I am especially grateful to Daniel Alvarez-Gavela, Oleg Lazarev, David Nadler, Sheel Ganatra, Vivek Shende, Laura Starkston and Kyler Siegel for contributing many ideas and suggestions for improvement of the current text. I am very grateful to the anonymous referee for critical remarks and many useful suggestions. Special thanks to Nikolai Mishachev for making the pictures.

\section{Weinstein hypersurfaces and Weinstein pairs}

Weinstein hypersurfaces are special cases of Liouville hypersurfaces introduced by Avdek in [3]. This and other related notions discussed in this paper are also similar to 
"stops" of Sylvan, [42] and Liouville sectors of Ganatra-Pardon-Shende, [26]. Related constructions are also considered in Ekholm-Lekili's paper [14].

\section{Weinstein hypersurfaces in a contact manifold}

Let $(Y, \xi)$ be a contact manifold. A codimension 1 submanifold $\Sigma \subset Y$ with boundary is called Weinstein hypersurface if there exists a contact form $\lambda$ for $\xi$ such that $\left(\Sigma,\left.\lambda\right|_{\Sigma}\right)$ is compatible with a Weinstein structure on $\Sigma$, i.e. $\left.d \lambda\right|_{\Sigma}$ is symplectic and the Liouville vector field $Z_{\Sigma}$ on $\Sigma$ dual to the Liouville form $\left.\lambda\right|_{\Sigma}$ is outward transverse to $\partial \Sigma$ and admits a Lyapunov function $\phi: \Sigma \rightarrow \mathbb{R}$. The Reeb vector field for $\lambda$ is transverse to $\Sigma$ and the boundary $\partial \Sigma$ of a Weinstein hypersurface $\Sigma$ is a codimension two contact submanifold of $(Y, \xi)$.

Though the induced Weinstein structure on $\Sigma$ depends on the choice of a contact form, its skeleton is independent of this choice. Indeed, the Liouville fields for the Liouville structures $\lambda$ and $f \lambda$ for a positive $f>0$ are proportional. In fact, as it is computed in Lemma 12.1 in [11] the form $f \lambda$ is Liouville if and only if $k:=$ $\inf (f+d f(Z))>0$, where $Z$ is the Liouville form for $\lambda$, and in that case the Liouville vector field for $f \lambda$ is equal to $\frac{1}{k} Z$. Moreover, the space of functions $f$ for which $f \lambda$ is Liouville (and hence in the considered case Weinstein) is contractible.

It follows that the skeleton $\operatorname{Core}\left(\Sigma,\left.\lambda\right|_{\Sigma}\right)$ is a stratified subset of $Y$ which consists of strata which are isotropic, and in the top dimension $n-1$ are Legendrian for the contact structure $\xi$.

Example 2.1. (i) Weinstein thickening of a Legendrian submanifold. Let $\Lambda \subset$ $(Y, \xi)$ be a Legendrian submanifold. Then it admits a Darboux neighborhood $U(\Lambda)$ isomorphic to $\left(J^{1}(\Lambda), d z-p d q\right), q \in \Lambda,\|p\|^{2}+z^{2} \leq \varepsilon^{2}$. Then $\Sigma(\Lambda):=U(\Lambda) \cap\{z=0\}$ is a Weinstein hypersurface symplectomorphic to the cotangent ball bundle of $\Lambda$. Up to Weinstein isotopy the Weinstein thickening $\Sigma(\Lambda)$ is independent of all the choices. ${ }^{2}$

(ii) Pages of open books. According to Giroux's theorem [25], any contact manifold admits an open book decomposition whose pages are Weinstein hypersurfaces.

(iii) Halves of convex hypersurfaces. Recall that a hypersurface $\Sigma$ in a contact manifold is called convex if it admits a transverse contact vector field, see [20, 24]. The set $D$ of points where the contact vector field is tangent to the

\footnotetext{
${ }^{2}$ Warning: unlike the case of a Legendrian isotopy, an isotopy of Weinstein hypersurfaces does not extend in general to an ambient contact diffeotopy.
} 
contact plane field, called a dividing set, is generically a smooth hypersurface which divides $\Sigma$ into two Liouville manifolds. In many interesting examples these Liouville manifolds are, in fact, Weinstein, and hence serve a rich source of Weinstein hypersurfaces.

Given two Legendrian isotopic submanifolds $\Lambda_{0}, \Lambda_{1} \subset(Y, \xi)$ their Weinstein thickenings $\Sigma\left(\Lambda_{0}\right)$ and $\Sigma\left(\Lambda_{1}\right)$ are isotopic as Weinstein hypersurfaces.

Problem 2.2. Is the converse true?

Here by isotopy we mean an isotopy of unparameterized submanifolds.

Note that an isotopy of Weinstein hypersurfaces carries $\Lambda_{0}$ to an exact Lagrangian submanifold $\widetilde{\Lambda}_{1} \subset \Sigma\left(\Lambda_{1}\right)$. Moreover, there is a symplectomorphism $\psi: \Sigma\left(\Lambda_{1}\right) \rightarrow$ $\Sigma\left(\Lambda_{1}\right)$ such that $\psi\left(\widetilde{\Lambda}_{1}\right)=\Lambda_{1}$. Hence, the positive answer to Problem 2.2 would follow from the positive resolution of the following special case of the nearby Lagrangian conjecture: Lagrangians which are images of the 0-section under a global symplectomorphism are Hamiltonian isotopic to the 0-section.

If the contact manifold $(Y, \xi)$ is symplectically fillable then one can prove that the Legendrian algebras $L H A\left(\Lambda_{0}\right)$ and $L H A\left(\Lambda_{1}\right)$ are isomorphir ${ }^{3}$. It is likely that this claim could be generalized to the case of a general contact manifold $(Y, \xi)$.

Problem 2.3. Is there an analog of the Legendrian algebra $L H A(\Lambda)$ for a general Weinstein hypersurface?

Let us return to the case of the Legendrian homology algebra of a Legendrian submanifold $\Lambda$ and pick a contact form $\lambda$ such that its Reeb vector field is tangent to the contact submanifold $\Delta:=\partial \Sigma(\Lambda)$. We also choose an almost complex structure $J$ on $\xi$ such that $\xi \cap T(\Delta)$ are $J$-invariant. This allows us to define a deformation $(A[t], D)$ of the Legendrian differential algebra $(A, \partial):=L H A(\Lambda)$ as follows. For a generating chord $c \in A$ define $D(c)=\sum_{k \geq 0}\left(\partial_{k} c\right) t^{k}$, where $\partial_{0}=\partial$ and $\partial_{k} c$ counts holomorphic curves with the intersection index $k$ with the symplectization of $\Delta$. This symplectization is a complex hypersurface in the symplectization of $Y$, and hence $k \geq 0$. The sum defining differential $D$ is finite due to the Gromov compactness.

Problem 2.4. Explore whether the above construction yields a genuinely new invariant of a Legendrian submanifold.

\footnotetext{
${ }^{3}$ I thank Sheel Ganatra and Tobias Ekholm for the discussion of this problem.
} 
Given a Weinstein hypersurface $\Sigma \subset Y$ we slightly extend it to a larger Weinstein hypersurface $\widetilde{\Sigma} \supset \Sigma$ such that on $\widetilde{\Sigma} \backslash \Sigma$ the Liouville form $\lambda$ can be written as $\left.t \lambda\right|_{\partial \Sigma}$, $t \in[1,1+\varepsilon]$. The extended hypersurface $\widetilde{\Sigma}$ has a neighborhood $\widetilde{U}$ diffeomorphic to $\widetilde{\Sigma} \times(-\varepsilon, \varepsilon)$ such that $\left.\lambda\right|_{\widetilde{U}}=\pi^{*}\left(\left.\lambda\right|_{\widetilde{\Sigma}}\right)+d u$ where $u$ is the coordinate corresponding to the second factor and $\pi$ the projection $\widetilde{U} \rightarrow \widetilde{\Sigma}$. Note that the level sets $\{u=$ const $\}$ are translates of $\widetilde{\Sigma}$ under the Reeb flow of the contact form $\lambda$. Pick a non-negative function $h: \widetilde{\Sigma} \rightarrow \mathbb{R}$ which is equal to 0 on $\Sigma$ and to $t-1$ near $\partial \widetilde{\Sigma}$ and set $U(\Sigma)=$ $U_{\varepsilon}(\Sigma):=\left\{h^{2}+u^{2} \leq \varepsilon^{2}\right\} \subset \widetilde{U}$. The neighborhood $U(\Sigma)$ will be called the contact surrounding of a Weinstein hypersurface $\Sigma$.

Proposition 2.5. Contact manifolds $Y \backslash \overline{U(\Sigma)}, Y \backslash \Sigma$ and $Y \backslash \operatorname{Core}\left(\Sigma,\left.\lambda\right|_{\Sigma}\right)$ are contactomorphic.

Let us first recall a few basic facts about convex hypersurfaces in contact manifolds. If a germ $\xi$ of a contact structure along a closed hypersurface $V$ in a $(2 n-1)$ dimensional manifold admits a transverse contact vector field $v$ then we canonically can construct a contact structure $\widehat{\xi}$ on $V \times \mathbb{R}$ which is invariant with respect to translations along the second factor and whose germ along any slice $V \times t, t \in \mathbb{R}$, is isomorphic to $\xi$. We will call $\widehat{\xi}$ the invariant extension of the convex germ $\xi$.

Lemma 2.6. Let $V$ be a closed $(2 n-2)$-dimensional manifold and $\xi$ a contact structure on $Y=V \times[0, \infty)$ which admits a contact vector field $v$ inward transverse to $V \times 0$ and such that its trajectories intersecting $V \times 0$ fill the whole manifold $Y$ (we do not require $v$ to be complete). Then $(Y, \xi)$ is contactomorphic to $(V \times[0, \infty), \widehat{\xi})$, where $\widehat{\xi}$ is the invariant extension of the germ of $\xi$ along $V \times 0$. Moreover, for any compact set $C \subset Y$, Int $C \supset V \times 0$, there exists a contactomorphism $h:(Y, \xi) \rightarrow(V \times[0, \infty), \widehat{\xi})$ which is equal to the identity on $V \times 0$ and which sends the contact vector field $\left.v\right|_{C}$ to the vector field $\frac{\partial}{\partial t}$.

Proof. It is sufficient to construct a complete contact vector field $\widetilde{v}$ on $V \times[0,1)$ which coincides with $v$ on $C$ and whose trajectories intersecting $V \times 0$ fill the whole manifold $V \times[0,1)$. We will construct it using the following inductive process. Take a sequence of compact sets $C_{0}=C, C_{1}, \ldots$, which exhausts $Y$, i.e. $\bigcup_{0}^{\infty} C_{j}=Y$ and $C_{j} \subset \operatorname{Int} C_{j}, j=0,1, \ldots$ Let $v_{1}$ be a contact vector field obtained by cutting off $v$ outside $C_{0}$ but inside $C_{1}$. Let $h_{1}$ be the time $T_{1}>1$ flow map of $v_{1}$, where $T_{1}$ is chosen sufficiently large to ensure that $h_{1}(V \times 0) \subset C_{1} \backslash C_{0}$. Denote by $\widetilde{C}_{0}$ the domain bounded by $V \times 0$ and $h_{1}(V \times 0)$ and by $\widetilde{v}_{1}$ the contact vector field equal to $v_{1}$ on $\widetilde{C}_{0}$ and to the push-forward vector field $\left(h_{1}\right)_{*} v$ on $Y \backslash \widetilde{C}_{0}$. Let $v_{2}$ be a contact 
vector field obtained by cutting off $\widetilde{v}_{1}$ outside $C_{1}$ but inside $C_{2}$ and denote by $h_{2}$ the time $T_{2}>T_{1}+1$ flow of $v_{2}$, where $T_{2}$ is chosen such that $h_{2}(V \times 0) \subset C_{2} \backslash C_{1}$. Denote by $\widetilde{C}_{1}$ the domain bounded by $V \times 0$ and $h_{2}(V \times 0)$ and by $\widetilde{v}_{2}$ the contact vector field equal to $v_{2}$ on $\widetilde{C}_{1}$ and to the push-forward vector field $\left(h_{1}\right)_{*} \widetilde{v}_{1}$ on $Y \backslash \widetilde{C}_{1}$. Continuing this process we construct a sequence of contact vector fields $\widetilde{v}_{1}, \widetilde{v}_{2}, \ldots$, which stabilize on compact sets $C_{1}, C_{2}, \ldots$ and converge to the contact vector field $\widetilde{v}$ on $Y$ with the required properties.

Proof of Proposition 2.5. The contact vector field $v=-Z_{\Sigma}-u \frac{\partial}{\partial u}$ is transverse to $\partial U(\sigma)$ and retracts $U(\Sigma)$ to $\operatorname{Core}\left(\Sigma, \lambda_{\Sigma}\right)$, and hence the contact structure on $U(\Sigma) \backslash$ $\operatorname{Core}\left(\Sigma, \lambda_{\Sigma}\right)$ is canonically isomorphic to $\partial U(\Sigma) \times[0, \infty)$ endowed with the invariant extension $\widehat{\xi}$ of the germ of contact structure $\xi$ along $\partial U(\Sigma)$. On the other hand, $v$ is transverse to $\partial U_{\delta}(\sigma)$ for each $\delta \leq \varepsilon$ and $\bigcup_{\delta \in(0, \varepsilon]} \partial U_{\delta}(\Sigma)=U(\Sigma) \backslash \Sigma$. Hence, applying Lemma 2.6 we conclude that $(U(\Sigma) \backslash \Sigma, \xi)$ is contactomorphic to $(\partial U(\Sigma) \times[0, \infty), \widehat{\xi})$, and the claim follows.

Remark 2.7. One of the corollaries of Lemma 2.6 is that any open domain in the standard contact $\left(\mathbb{R}^{2 n+1}, d z+\sum x_{i} d y i-y_{i} d x_{i}\right)$ which is star-shaped with respect to the contact vector field $2 z \frac{\partial}{\partial z}+\sum x_{i} \frac{\partial}{\partial x_{i}}+y_{i} \frac{\partial}{\partial y_{i}}$ is contactomorphic to $\mathbb{R}^{2 n+1}$. On the other hand, in the standard contact $\mathbb{R}^{3}$ any open domain diffeomorphic to $\mathbb{R}^{3}$ is contactomorphic to $\mathbb{R}^{3}$, see [17].

Problem 2.8. Is there a domain in the standard contact $\mathbb{R}^{2 n+1}, n>2$, which is diffeomorphic to the closed ball, has convex in contact sense boundary, but whose interior is not contactomorphic to the standard $\mathbb{R}^{2 n+1}$ ? Or even are there any open domains in the standard contact $\mathbb{R}^{2 n+1}, n>2$, which are diffeomorphic but not contactomorphic to $\mathbb{R}^{2 n+1}$ ?

\section{Weinstein pairs}

A Weinstein pair $(\mathfrak{W}, \Sigma)$ consists of a Weinstein domain $\mathfrak{W}=(X, \lambda, \phi)$ together with a Weinstein hypersurface $\left(\Sigma,\left.\lambda\right|_{\Sigma}\right)$ in its boundary $\partial X$. Equivalently, a Weinstein pair can be viewed as a Weinstein manifold with cylindrical end, together with a Weinstein hypersurface in its ideal contact boundary. 
Let $\Lambda=\operatorname{Core}(\Sigma)$ be the skeleton of $\Sigma$ and

$$
\widehat{\Lambda}:=\bigcup_{t \geq 0} Z^{-t}(\Lambda)
$$

be its saturation by the trajectories of the Liouville vector field $Z$. The union

$$
\operatorname{Core}(\mathrm{X}, \Sigma):=\operatorname{Core}(\mathrm{X}) \cup \widehat{\Lambda}
$$

is called the core, or the skeleton of the Weinstein pair.

It turns out that it is possible to modify the Liouville form $\lambda$ on $X$ in a neighborhood of $\Sigma$ in $X$ to make the attractor of the modified Liouville vector field equal to the skeleton $\operatorname{Core}(\mathrm{X}, \Sigma)$.

Given a Weinstein pair $(\mathfrak{W}, \Sigma), \mathfrak{W}=(X, \omega, \lambda, Z, \phi)$, let $U=U(\Sigma) \subset \partial X$ be its contact surrounding. Denote by $Z_{\Sigma}$ the Liouville field dual to $\left.\lambda\right|_{\Sigma}$ and by $\phi_{\Sigma}$ its Lyapunov function. A Liouville form $\lambda_{0}$, the corresponding Liouville vector field $Z_{0}$ for $\omega$ on $X$ and a smooth function $\phi_{0}: X \rightarrow \mathbb{R}$ are called adjusted to the structure of the pair if (see Fig. 2.1)

- $Z_{0}$ is tangent to $\partial X$ on $U(\Sigma)$ and transverse to $\partial X$ elsewhere;

- $\left.Z_{0}\right|_{U(\Sigma)}=Z_{\Sigma}+u \frac{\partial}{\partial u}$

- the attractor $\bigcap_{t \geq 0} Z_{0}^{-t}(X)$ of the Liouville vector field $-Z_{0}$ coincides with the core Core $(\mathrm{X}, \Sigma)$ of the Weinstein pair;

- the function $\phi_{0}: X \rightarrow \mathbb{R}$ is Lyapunov for $Z_{0}$ and such that $\left.\phi_{0}\right|_{U(\Sigma)}=\phi_{\Sigma}+u^{2}$ and $\phi_{0}$ has no critical values $\geq \varepsilon^{2}=\left.\phi_{0}\right|_{\partial U(\Sigma)}$.

Proposition 2.9. Given a Weinstein pair $(\mathfrak{W}, \Sigma)$, $\mathfrak{W}=(X, \lambda, \phi)$, there exist a Liouville form $\lambda_{0}$ for $\omega$ and a function $\phi_{0}: X \rightarrow \mathbb{R}$ such that

- $\lambda_{0}, \phi_{0}$ are adjusted to $(\mathfrak{W}, \Sigma)$;

- $\lambda_{0}$ coincides with $\lambda$ outside a neighborhood of $\Sigma$;

Moreover, there exists an extension $\widetilde{\lambda}, \widetilde{\phi}$ of $\left(\lambda_{0}, \phi_{0}\right)$ to a slightly bigger domain $\widetilde{X} \supset X$ such that the $\widetilde{\mathfrak{W}}:=(\widetilde{X}, \widetilde{\lambda}, \widetilde{\phi})$ is a Weinstein domain and Core $(\widetilde{\mathfrak{W}})=\operatorname{Core}(\mathfrak{W}, \Sigma)$. 
To construct the adjusted Liouville field $Z_{0}$ let us write the form $\lambda$ near $\partial X$ as $s\left(d u+\lambda_{\Sigma}\right)$ near $U(\Sigma)$. Note that the Hamiltonian vector field $Y$ for a function su near $U(\Sigma)$ coincides with $-s \frac{\partial}{\partial s}+u \frac{\partial}{\partial u}+Z_{\Sigma}$, and hence by appropriately cutting off the function su outside a neighborhood of $U(\Sigma)$ and subtracting the differential $d g$ of the resulting function $g$ to the Liouville form $\lambda$ we get the Liouville form $\lambda_{0}$ with the required properties. Note that the form $\left.\lambda_{0}\right|_{U}$ is no more contact. Instead, $\left.\lambda_{0}\right|_{U}=\pi^{*}\left(\left.\lambda\right|_{\Sigma}\right)$.

Suppose that $\lambda_{0}, \phi_{0}$ are adjusted to the Weinstein pair $(\mathfrak{W}, \Sigma)$. Recall that $\left.\phi_{0}\right|_{\partial U(\Sigma)}=$ $\varepsilon^{2}$. Denote $X_{0}=\left\{\phi_{0} \leq \varepsilon^{2}\right\}$. We note that $\phi_{0}$ has no critical points in $X \backslash \operatorname{Int} X_{0}$, and hence $X_{0}$ is a manifold with boundary with a corner along $\partial U(\Sigma)$ which is homeomorphic to $X$. We will sometime refer to $\left(X_{0}, \lambda_{0}, \phi_{0}\right)$ as the cornered version of the Weinstein pair $(\mathfrak{W}, \Sigma) \mathbb{4}^{4}$ For instance, the cornered version of the standard Weinstein ball $B^{2 n}$ is the cotangent ball bundle of $D^{n}$. Thus, it is always possible to go back and forth between the original and adjusted (cornered) versions of a Weinstein pair, and we will be using the term "Weinstein pair" for both versions.
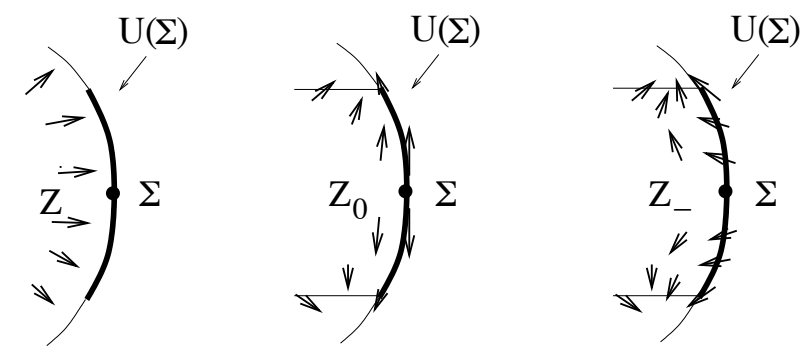

Fig. 2.1: Modifications of a Weinstein pair structure.

Remark 2.10. There are several other useful adjustments of a Weinstein pair structure. Ekholm and Lekili in [14], Section B.3, are doing a similar to the cornered version construction by deforming the boundary $\partial X$ near $U(\Sigma)$ without changing $Z$, as on Fig. 2.2. Without defining here Sylvan's stop structure we just say that for a given Weinstein pair there is a contractible space of choices of stop structures on the completion.

One can also transform a Weinstein pair into a Weinstein cobordism whose negative boundary is $U(\Sigma)$, see Fig. 2.1.

\footnotetext{
${ }^{4}$ The completion of the cornered version of a Weinstein pair is a special case of a Liouville sector in the sense of [26].
} 
Let $\left(X_{0}, \lambda_{0}, Z_{0}, \phi_{0}\right)$ be the cornered adjusted version of a Liouville pair structure $(\mathfrak{W}, \Sigma)$, as in Proposition 2.9. There exists a Liouville form $\lambda_{-}$on $X_{0}$ such that $\left(X_{0}, \lambda_{-}, \phi_{0}\right)$ is a sutured Weinstein cobordism structure with $\partial_{-} X_{0}=U(\Sigma)$, and $\operatorname{Core}\left(\mathrm{X}_{0}, \lambda_{-}, \phi_{0}\right)=\operatorname{Core}(\mathfrak{W}, \Sigma)$.

To obtain such a form $\lambda_{-}$one subtracts from $\lambda$ the differential of the appropriately cut off function $2 s u$ instead of the function $s u$ used to modify $\lambda$ into $\lambda_{0}$.

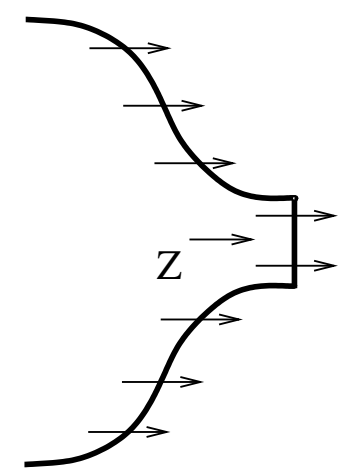

Fig. 2.2: Ekholm-Lekili deformation of $\partial X$.

\section{Operations on Weinstein pairs}

\subsection{Splitting and gluing of Weinstein pairs}

Let $\mathfrak{W}=(X, \lambda, Z, \phi)$ be a Weinstein domain. A hypersurface $(P, \partial P) \subset(X, \partial X)$ is called splitting for $\mathfrak{W}$ if it satisfies the following conditions:

- $\partial P$ splits the boundary $\partial X$ into two parts, $\partial X=Y_{-} \cup Y_{+}$with $\partial Y_{-}=\partial Y_{+}=$ $Y_{+} \cap Y_{-}=\partial P$ (and respectively, $P$ divides $X$ into two parts $X_{+}$and $X_{-}$with $\partial X_{-}=P \cup Y_{-}, \partial X_{+}=P \cup Y_{+}$and $X_{+} \cap X_{-}=P$;

- the Liouville vector field $Z$ is tangent to $P$;

- there exists a hypersurface $(S, \partial S) \subset(P, \partial P)$ which is Weinstein for the restricted Liouville form $\left.\lambda\right|_{S}$, tangent to the vector field $Z$ and intersects all leaves of the characteristic foliation $\mathcal{F}$ of the hypersurface $P$; we will refer to $S$ as the Weinstein soul of the splitting hypersurface $P$ and denote it by $\operatorname{Soul}(P)$. 
Note that the latter condition together with Lemma 2.6 imply that $P$ is contactomorphic to the contact surrounding of its Weinstein soul.

It follows that $\left(S,\left.\lambda\right|_{S},\left.\phi\right|_{S} ; \partial S\right)$ is a codimension two Weinstein subdomain of $X$ and Core $\left(\mathrm{S},\left.\lambda\right|_{\mathrm{S}},\left.\phi\right|_{\mathrm{S}}\right)=\operatorname{Core}(\mathfrak{W}) \cap \mathrm{P}$. Moreover, $\left(\mathfrak{W}_{ \pm} ; S\right)$, where $\mathfrak{W}_{ \pm}:=\left(X_{ \pm},\left.\lambda\right|_{X_{ \pm}},\left.\phi\right|_{X_{ \pm}}\right)$ are cornered Weinstein pairs and $\operatorname{Core}\left(\mathfrak{W}_{ \pm} ; \mathrm{S}\right)=\operatorname{Core}(\mathfrak{W}) \cap \mathrm{X}_{ \pm}$.

The gluing construction reverses the splitting. This operation was considered by Avdek in [3] in the context of Liouville hypersurfaces. Let $(\mathfrak{W}, \Sigma)$ and $\left(\mathfrak{W}^{\prime}, \Sigma^{\prime}\right)$ be two Weinstein pairs and $\left(X_{0}, \lambda_{0}, \phi_{0}\right),\left(X_{0}^{\prime}, \lambda_{0}^{\prime}, \phi_{0}^{\prime}\right)$ their cornered forms. Let $F$ : $\left(\Sigma,\left.\lambda\right|_{\Sigma},\left.\phi\right|_{\Sigma}\right) \rightarrow\left(\Sigma^{\prime},\left.\lambda^{\prime}\right|_{\Sigma^{\prime}},\left.\phi^{\prime}\right|_{\Sigma^{\prime}}\right)$ be a Weinstein isomorphism. We extend $F$ to a contactomorphism $U(\Sigma) \rightarrow U\left(\Sigma^{\prime}\right)$, still denoted by $F$, and use it to define a domain

$$
X \underset{F}{\sqcup} X^{\prime}:=X_{0} \sqcup X_{0}^{\prime} /\left\{(x \in U(\Sigma)) \sim\left(F(x) \in U\left(\Sigma^{\prime}\right)\right) .\right.
$$

Then the Liouville forms $\lambda_{0}$ and $\lambda_{0}^{\prime}$, as well as Lyapunov functions $\phi_{0}: X_{0} \rightarrow \mathbb{R}$ and $\phi_{0}^{\prime}: X_{0}^{\prime} \rightarrow \mathbb{R}$, can be glued together to define a Weinstein structure $(\mathfrak{W}, \Sigma) \underset{F}{\cup}\left(\mathfrak{W}^{\prime}, \Sigma^{\prime}\right):=$ $\left(X_{F}, \lambda_{F}, \phi_{F}\right)$, see Fig. 3.1 .

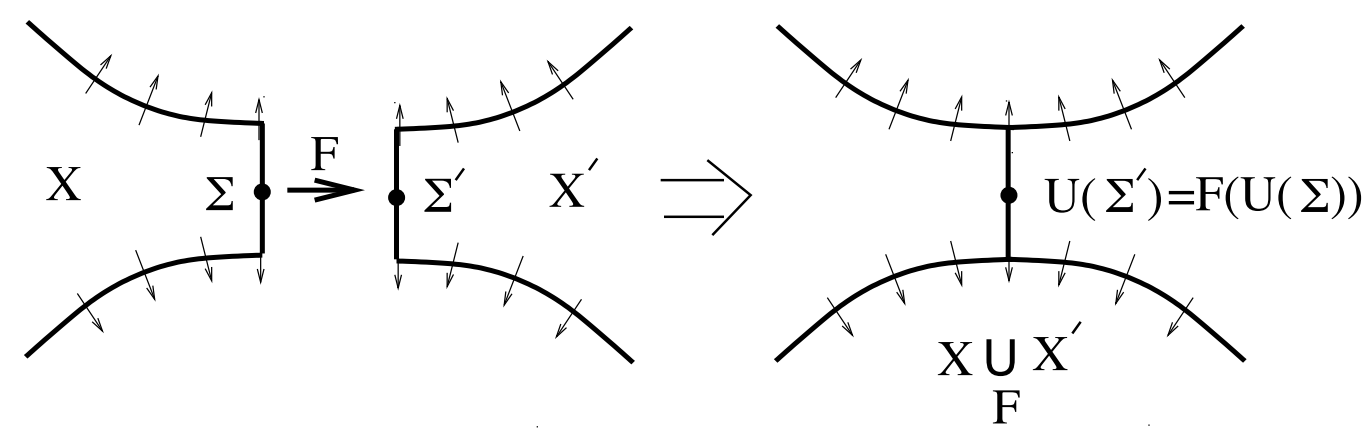

Fig. 3.1: Gluing of Weinstein pairs.

Note that

$$
\operatorname{Core}\left(\mathrm{X}_{\mathrm{F}}, \lambda_{\mathrm{F}}, \phi_{\mathrm{F}}\right)=\operatorname{Core}(\mathrm{X}, \Sigma) \underset{\left.\mathrm{F}\right|_{\operatorname{Core}(\Sigma)}}{\cup} \operatorname{Core}\left(\mathrm{X}^{\prime}, \Sigma^{\prime}\right)
$$

Note that the constructed Weinstein domain $X_{F}$ contains $U(\Sigma)$ as its splitting hypersurface. Applying the above described splitting construction we get back the Weinstein pairs $(\mathfrak{W}, \Sigma)$ and $\left(\mathfrak{W}^{\prime}, \Sigma^{\prime}\right)$. 
The gluing of Weinstein pairs is a generalization of the Legendrian surgery construction (or rather Weinstein handle attachment). When $\Sigma=\Sigma(\Lambda)$ for a Legendrian $\Lambda \subset \partial X, X^{\prime}=B^{2 n}$ and $\Sigma^{\prime}=\Sigma\left(\Lambda_{0}\right)$, where $\Lambda_{0}$ is the Legendrian unknot in $S^{2 n-1}=\partial B$, then $\left(X_{F}, \lambda_{F}, \phi_{F}\right)$ is the Weinstein $n$-handle attachment to $X$ along $\Lambda$. Conversely, the general gluing operation $(\mathfrak{W}, \Sigma) \cup_{F}\left(\mathfrak{W}^{\prime}, \Sigma^{\prime}\right)$ can be decomposed into a sequence of subcritical and critical handle attachments. To do that, one fixes first a Weinstein handle decomposition of $\Sigma$, and then for each handle of index $k$ of this decomposition one needs to attach a handle of index $k+1$ to the glued domains. For instance, for a handle of index 0 centered at a point $p \in \Sigma$ one attaches a handle of index 1 along an arc connecting the point $p \in \Sigma$ with its image $p^{\prime}=F(p) \in \Sigma^{\prime}$ under the gluing map.

Both, splitting and gluing constructions can be naturally generalized to the relative setting. Let $(\mathfrak{W}, \Sigma)$ and $\left(\mathfrak{W}^{\prime}, \Sigma^{\prime}\right)$ be two Weinstein pairs. Suppose that $\Sigma$ and $\Sigma^{\prime}$ are split by splitting hypersurfaces $T \subset \Sigma$ and $T^{\prime} \subset \Sigma^{\prime}$ as $\Sigma=\Sigma_{-} \cup \Sigma_{+}$ and $\Sigma^{\prime}=\Sigma_{-}^{\prime} \cup \Sigma_{+}^{\prime}$ and we are given a Weinstein isomorphism $F: \Sigma_{+} \rightarrow \Sigma_{-}^{\prime}$. Then the result of the partial gluing is the pair $(\mathfrak{W}, \Sigma) \underset{F, T, T^{\prime}}{\cup}\left(\mathfrak{W}^{\prime}, \Sigma^{\prime}\right)$ which consists of the Weinstein domain $\left(\mathfrak{W}, \Sigma_{-}\right) \bigcup_{F}\left(\mathfrak{W}^{\prime}, \Sigma_{+}^{\prime}\right)$ together with the Weinstein hypersurface $\Sigma_{-} \cup_{F \mid \operatorname{Soul}(T)} \Sigma_{+}^{\prime} \subset X \cup_{F} X^{\prime}$ which is the result of gluing the Weinstein pairs $\left(\Sigma_{+}, \operatorname{Soul}(T)\right)$ and $\left(\Sigma_{-}^{\prime}, \operatorname{Soul}\left(T^{\prime}\right)\right)$ using the Weinstein isomorphism $\left.F\right|_{\operatorname{Soul}(T)}$.

The reverse operation to the partial gluing of Weinstein pairs is a splitting of a Weinstein pair $(\mathfrak{W}, \Sigma), \mathfrak{W}=(X, \lambda, \phi)$, along a splitting hypersurface $(P, Q:=\partial P) \subset$ $(X, \partial X)$ for the Weinstein domain $X$ where in addition $P$ satisfies the following condition:

- $Q$ intersects $\Sigma$ transversely, $Q \cap \Sigma=\operatorname{Soul}(Q)$ and $Q \cap \Sigma$ is a splitting hypersurface for $\Sigma$, which splits it into $\Sigma_{+}$and $\Sigma_{-}$;

The result of this splitting are two Weinstein pairs $\left(X_{-}, \widetilde{\Sigma}_{-}\right)$and $\left(X_{+}, \widetilde{\Sigma}_{+}\right)$, where the Weinstein hypersurface $\widetilde{\Sigma}_{ \pm} \subset \partial X_{ \pm}=Y_{ \pm} \cup P$ is the result of gluing of Weinstein pairs $\left(\Sigma_{ \pm}, \operatorname{Soul}(Q \cap \Sigma)\right)$ and $(\operatorname{Soul}(P), \operatorname{Soul}(Q \cap \Sigma))$.

As in the absolute case, the gluing operation of Weinstein pairs glues their skeleta along the skeleta of glued hypersurfaces. Conversely, a splitting of the skeleton of a Weinstein domain lifts to a splitting of a Weinstein domain into two Weinstein pairs. 


\subsection{Product and Stabilization of Weinstein pairs}

Given two Weinstein pairs $(\mathfrak{W}, \Sigma)$ and $\left(\mathfrak{W}^{\prime}, \Sigma^{\prime}\right)$, where $\mathfrak{W}=(X, \lambda, \phi), \mathfrak{W}^{\prime}=\left(X^{\prime}, \lambda^{\prime}, \phi^{\prime}\right)$ we define their product as the Weinstein pair

$$
(\mathfrak{W}, \Sigma) \times\left(\mathfrak{W}^{\prime}, \Sigma^{\prime}\right):=\left(X \times X^{\prime}, \lambda \oplus \lambda^{\prime},\left(\Sigma \times X^{\prime} ; \Sigma \times \Sigma^{\prime}\right) \underset{\mathrm{Id}}{\bigsqcup}\left(X \times \Sigma^{\prime}, \Sigma \times \Sigma^{\prime}\right)\right) .
$$

Here $\left.\left(\Sigma \times X^{\prime} ; \Sigma \times \Sigma^{\prime}\right) \underset{\text { Id }}{\bigsqcup}\left(X \times \Sigma^{\prime}, \Sigma \times \Sigma^{\prime}\right)\right)$ is the result of gluing of two Weinstein pairs by the identity map between the Weinstein hypersurfaces $\Sigma \times \Sigma^{\prime} \subset \partial\left(X \times \Sigma^{\prime}\right)$ and $\Sigma \times \Sigma^{\prime} \subset \partial\left(\Sigma \times X^{\prime}\right)$. We note that

$$
\text { Core }\left((\mathfrak{W}, \Sigma) \times\left(\mathfrak{W}^{\prime}, \Sigma^{\prime}\right)\right)=\operatorname{Core}(\mathfrak{W}, \Sigma) \times \operatorname{Core}\left(\mathfrak{W}^{\prime}, \Sigma^{\prime}\right) .
$$

In the case when $\left(X^{\prime}, \Sigma^{\prime}\right)$ is the Weinstein pair $\left(T^{*} D^{k}, T^{*} S^{k-1}\right)$ the product operation is called the stabilization (or $k$-stabilization). It was first proposed in a slightly different form by M. Kontsevich, [28]. The core of the $k$-stabilized pair $(\mathfrak{W}, \Sigma)$ is equal to $\operatorname{Core}(\mathfrak{W}, \Sigma) \times D^{\mathrm{k}}$.

It is important to stress the point that the result of the stabilization is always a Weinstein pair with a non-empty hypersurface in the boundary, even if we begin with the absolute case of a Weinstein domain.

\subsection{Weinstein homotopy as a Weinstein pair}

Consider a Weinstein structure $\mathfrak{W}_{0}:=\left(X, \omega, \lambda_{0}, \phi_{0}\right)$ and its 1-stabilization $\mathfrak{W}^{\text {st }}:=$ $\mathfrak{W} \times T^{*} I$, viewed as a Weinstein pair $\left(X \times T^{*} I, \lambda_{0}+u d t, X \times 0 \cup X \times 1\right)$. Consider a Weinstein homotopy $\mathfrak{W}_{t}:=\left(X, \lambda_{t}=\lambda_{0}+d h_{t}, \phi_{t}\right), t \in[0,1]$. We assume, in addition, that $\dot{h}_{1}=\dot{h}_{0}=0$, where we denoted $\dot{h}_{t}:=\frac{d h_{t}}{d t}(t)$. This condition can always be arranged by a re-parameterization of the homotopy. Consider the product $X \times T^{*} I$ with the symplectic form $\Omega:=\omega \oplus d u \wedge d t$, where $(u, t)$ are canonical coordinates on $T^{*} I$ (so that $u=0$ defines the 0 -section). Note that the 1 -form $\widetilde{\lambda}:=\lambda_{t}+\left(u+\dot{h}_{t}\right) d t$ is a Liouville form for $\Omega$. Indeed, $d \widetilde{\lambda}:=d \lambda_{t}+d t \wedge d \dot{h}_{t}+d \dot{h}_{t} \wedge d t+d u \wedge d t=\omega+d u \wedge d t$. We have $\left.\widetilde{\lambda}\right|_{X_{0}}=\lambda_{0}$ and $\left.\widetilde{\lambda}\right|_{X_{1}}=\lambda_{1}$.

Proposition 3.1. There exists a function $\widetilde{\phi}=X \times T^{*} I \rightarrow \mathbb{R}$ such that

$$
\begin{aligned}
& \left(\left(X \times T^{*} I, \tilde{\lambda}:=\lambda_{t}+\left(u+\dot{h}_{t}\right) d t, \widetilde{\phi} ; X_{0} \cup X_{1}\right)\right. \\
& \text { where } X_{0}:=X \times\{t=u=0\}, ; X_{1}:=X \times\{t=1, u=0\},
\end{aligned}
$$

is a Weinstein pair. 
We call this pair the concordance generated by the homotopy $\mathfrak{W}_{t}$.

Proof. Note that the corresponding to $\widetilde{\lambda}$ Liouville vector field is given by the formula $\widetilde{Z}=Z_{t}+\left(u+\dot{h}_{t}\right) \frac{\partial}{\partial u}$, where $Z_{t}$ is the Liouville vector field corresponding to $\lambda_{t}$. Define the function $\widetilde{\phi}$ by the formula $\widetilde{\phi}=\phi_{t}+\frac{k}{2}\left(u+\dot{h}_{t}\right)^{2}$, where a positive constant $k$ will be chosen later. Then we have

$$
d \widetilde{\phi}(\widetilde{Z})=d \phi_{t}\left(Z_{t}\right)+k\left(u+\dot{h}_{t}\right)^{2}+k\left(u+\dot{h}_{t}\right) d \dot{h}_{t}\left(Z_{t}\right) .
$$

Not that $\left|d \phi_{t}\left(Z_{t}\right) \geq a\right| \mid Z_{t} \|^{2}$ and $\left|d \dot{h}_{t}\left(Z_{t}\right)\right| \leq b\left\|Z_{t}\right\|$ for some constants $a, b>0$. Denoting $X:=\left\|Z_{t}\right\|, Y:=u+\dot{h}_{t}$ we can write

$$
\begin{aligned}
& |d \widetilde{\phi}(\widetilde{Z})| \geq a|| Z_{t} \|^{2}+k\left(u+\dot{h}_{t}\right)^{2}-b k\left|u+\dot{h}_{t}\right||| Z_{t}|| \\
& a X^{2}+k Y^{2}-b k X Y .
\end{aligned}
$$

The quadratic form $a X^{2}+k Y^{2}-b k X Y$ is positive definite if $b^{2} k^{2}-4 a k<0$ or $k<\frac{4 a}{b^{2}}$. Under this condition, which can be arranged by choosing the constant $k$ sufficiently small, we get $|d \widetilde{\phi}(\widetilde{Z})| \geq c\left(X^{2}+Y^{2}\right) \geq \widetilde{c}|| \widetilde{Z} \|^{2}$ for positive constants $c, \widetilde{c}$. This concludes the proof.

Remark 3.2. The critical point locus of $\widetilde{\phi}$ (= the zero locus of $\widetilde{Z}$ ) is equal to

$$
\widetilde{C}=\left\{(x, t, u) ; x \text { is a critical point of } \phi_{t}, u=\dot{h}_{t}(x), t \in[0,1]\right\} .
$$

The stable manifold of a critical point $\left(x_{0}, t_{0}, u_{0}\right)$ projects to the stable manifold of the critical point $x_{0}$ of $\phi_{t_{0}}$. Its $u$-coordinate can be found by solving the inhomogeneous linear ODE

$$
\frac{d u(\gamma(s))}{d s}=u(\gamma(s))+\dot{h}_{t_{0}}(\gamma(s))
$$

with the asymptotic boundary condition $\lim _{s \rightarrow \infty} u(\gamma(s))=u_{0}$, where $\gamma(s)$ is a trajectory of $X_{t_{0}}$ converging to the critical point $x_{0}$.

\section{Looseness and Flexibility}

Let us recall that in contact manifolds of dimension $2 n-1 \geq 5$ there is a local

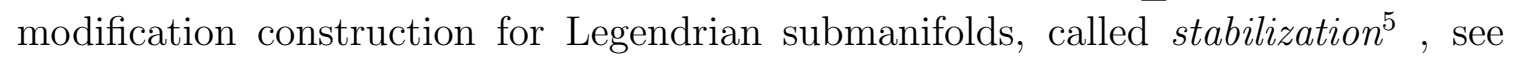
[16, 35, 11]. This operation can be performed in an arbitrarily small neighborhood

\footnotetext{
${ }^{5}$ The term "stabilization" is used here in a completely different sense than in Section 3.2 .
} 
of any point of a Legendrian. Moreover, it can also be performed without changing the formal Legendrian isotopy class of the Legendrian submanifold. In her 2012 paper [35] Emmy Murphy called a Legendrian submanifold loose if it is isotopic to a stabilization of another Legendrian submanifold, and showed that loose Legendrians satisfy an $h$-principle: any two loose formally isotopic Legendrians can be connected by a Legendrian isotopy.

The notion of flexibility, see [11, for Weinstein cobordisms is tightly related to the looseness property of Legendrian knots. One first defines flexibility for elementary Weinstein cobordisms, i.e. Weinstein cobordisms $(W, \omega, Z, \phi)$ without any $Z$-trajectories connecting critical points of the Lyapunov function $\phi$. An elementary $2 n$-dimensional, $n>2$, Weinstein cobordism $(W, \omega, X, \phi)$ is called flexible if the attaching spheres of all index $n$ handles form in $\partial_{-} W$ a loose Legendrian link (i.e. each sphere is loose in the complement of the others). A Weinstein structure is called flexible if it is homotopic to one which can be decomposed into elementary flexible cobordisms.

As it was shown by E. Murphy and K. Siegel in [37] existence of a decomposition into flexible elementary cobordisms really depends on the choice of a particular Weinstein structure in the given homotopy class. Moreover, there exist non-flexible Weinstein domains which become flexible after attaching an $n$-handle.

Flexible Weinstein structures are indeed flexible: they abide a number of $h$-principles.

Theorem 4.1. (i) ([1] ) Any two flexible Weinstein structures on a given smooth cobordism are homotopic as Weinstein structures provided that the corresponding symplectic forms are in the same homotopy class of non-degenerate (but not necessarily closed) 2-forms.

(ii) ([11]) Let $(X, \omega, Z, \phi)$ be any flexible Weinstein structure and $\phi_{t}, t \in[0,1]$, be a family of generalized Morse functions such that $\phi_{0}=\phi$. Then there exists a homotopy $\left(X, \omega_{t}, Z_{t}, \phi_{t}\right)$ of Weinstein structures.

(iii) ([22]) Let $\left(X_{ \pm}, \omega_{ \pm}, Z_{ \pm}, \phi_{ \pm}\right)$be two Weinstein structures. Suppose that the structure $\left(X_{-}, \omega_{-}, Z_{-}, \phi_{-}\right)$is flexible and that there exists an embedding $f: X_{-} \rightarrow$ $X_{+}$such that the forms $\omega_{-}$and $f^{*} \omega_{+}$are homotopic as non-degenerate (but not necessarily closed) 2-forms. Then there exists a homotopy of Weinstein structures $\left(X_{-}, \omega_{-}^{t}, Z_{-}^{t}, \phi_{-}^{t}\right), t \in[0,1]$, beginning with $\left(X_{-}, \omega_{-}^{0}=\omega_{-}, Z_{-}^{0}=\right.$ $\left.Z_{-}, \phi_{-}^{0}=\phi_{-}\right)$and an isotopy $f^{t}: X_{-} \rightarrow X_{+}$beginning with $f^{0}=f$ such that $\left(f^{1}\right)^{*} \omega_{+}=\omega_{-}^{1}$.

At first glance Theorem 4.1 implies that symplectic topology of flexible Weinstein 
manifolds is quite boring. This is also confirmed by the fact that symplectic homology in all its flavors of a flexible Weinstein manifold is trivial. However, as we will see below in Section 7 the contact boundaries of flexible Weinstein domains have a rich contact topology.

The looseness property of a Legendrian submanifold can be naturally extended to Weinstein hypersurfaces of contact manifolds. A Weinstein hypersurface $\Sigma$ of a contact manifold $Y$ of dimension $2 n+1 \geq 5$ is called loose if for each $n$-dimensional strata $S$ of the skeleton $\operatorname{Core}(\Sigma)$ there is a ball $B_{S} \subset Y \backslash(\operatorname{Core}(\Sigma) \backslash \mathrm{S})$ such that $B_{S} \cap S$ is loose in $B_{S}$ relative $\partial\left(B_{S} \cap S\right)$. A canonical Weinstein thickening of a loose Legendrian knot is loose. However, it is unclear whether looseness is preserved under Weinstein isotopy.

Problem 4.2. Is looseness property preserved under a Weinstein isotopy of $\Sigma$. In particular, suppose that a Weinstein thickening $\Sigma(\Lambda)$ of a Legendrian knot $\Lambda$ is isotopic in the class of Weinstein hypersurfaces to a loose Weinstein hypersurface. Does this imply that $\Lambda$ itself is loose?

Proposition 4.3. Let $(\mathfrak{W}, \Sigma)$ and $\left(\mathfrak{W}^{\prime}, \Sigma^{\prime}\right)$ where $\mathfrak{W}=(X, \lambda, \phi)$, $\mathfrak{W}^{\prime}=\left(X^{\prime}, \lambda^{\prime}, \phi^{\prime}\right)$, be two Weinstein pairs and

$$
(\mathfrak{W}, \Sigma) \times\left(\mathfrak{W}^{\prime}, \Sigma^{\prime}\right):=\left(X \times X^{\prime}, \lambda \oplus \lambda^{\prime}, \widetilde{\Sigma}:=\left(\Sigma \times X^{\prime} ; \Sigma \times \Sigma^{\prime}\right) \underset{\mathrm{Id}}{\sqcup}\left(X \times \Sigma^{\prime}, \Sigma \times \Sigma^{\prime}\right)\right)
$$

be their product. Suppose that $\Sigma$ is loose in $\partial X$. Then $\widetilde{\Sigma}$ is loose in $\partial\left(X \times X^{\prime}\right)$.

Indeed, this is straightforward from the following fact: given any contact manifold $(Y,\{\alpha=0\})$, a Liouville manifold $(U, \mu)$, a loose Legendrian $\Lambda \subset Y$ and a Lagrangian $L \subset U$ with $\left.\mu\right|_{L}=0$, then the Legendrian $\Lambda \times L \subset(Y \times U,\{\alpha \oplus \mu=0\})$ is loose as well.

Let us stress the point that while flexibility of a Weinstein manifold is its intrinsic property, the looseness of a Weinstein hypersurface depends on its embedding in the contact manifold. However, the above fact about the looseness of a product shows that flexibility always implies looseness (I thank the referee for this argiment).

Proposition 4.4. Let $(Y, \xi)$ be a contact manifold of dimension $\geq 7$, and $\Sigma \subset Y$ a flexible Weinstein hypersurface. Then $\Sigma$ is loose.

Indeed, let $\alpha$ be a contact form for $\xi$ which restricts to a Liouville form $\mu$ on $\Sigma$. Consider a Weinstein subdomain $\Sigma_{0} \subset \Sigma$ and let a Lagrangian disc $\Delta \subset \Sigma \backslash \Sigma_{0}$ be attached to $\Sigma_{0}$ along a loose Legendrian sphere $\Lambda:=\partial \Delta \subset \partial \Sigma_{0}$. In a neighborhood 
$U \supset \partial \Sigma_{0}$ in $\Sigma$ the Liouville form $\mu$ can be written as $s \beta, s \in(1-\varepsilon, 1+\varepsilon)$ for a contact form on $\partial \Sigma_{0}$, and on a neighborhood $\widetilde{U}$ of $U$ in $Y$ the contact form $\alpha$ can be written as $d t+s \beta=s(u d t+\beta),|t|<\varepsilon, u=\frac{1}{s}$. Hence, $\widetilde{U}$ can be viewed as the product of the contact manifold $\left(\partial \Sigma_{0}, \beta\right)$ and a Liouville subdomain

$$
Q:=\left\{(u, t) \in\left(-\frac{1}{1+\varepsilon}, \frac{1}{1-\varepsilon}\right) \times(-\varepsilon, \varepsilon)\right\} \subset\left(\mathbb{R}^{2}, u d t\right)
$$

while $\left.\Delta \cap \widetilde{U}=\Lambda \times\left\{t=0, \frac{1}{1-\varepsilon}<u \leq 1\right]\right\} \subset \Sigma_{0} \times Q$. Hence looseness of attaching spheres of top index Weinstein handles of $\Sigma$ implies looseness of their Lagrangian cores viewed as Legendrian submanifolds of $Y$.

The notion of flexibility naturally extends to Weinstein pairs. A Weinstein pair $(\mathfrak{W}=(X, \lambda, \phi), \Sigma)$ is called flexible if it is flexible viewed as a cobordism between $\partial X_{-}=U(\Sigma)$ and $\partial_{+} X=X \backslash \operatorname{Int} U(\Sigma)$, see Remark 2.10. It is straightforward to see that flexibility is preserved under the stabilization construction. However, the converse is not clear.

Problem 4.5. Suppose that the stabilization of a Weinstein pair is flexible. Does this imply that the Weinstein pair itself is flexible? More generally, does existence of a homotopy between stabilizations of two Weinstein (pair) structures implies existence of a homotopy between the structures themselves?

Attaching a critical handle along a loose Legendrian knot to a flexible Weinstein domain by definition preserves its flexibility. This generalizes to the following

Proposition 4.6. Let $(\mathfrak{W}=(X, \lambda, \phi), \Sigma)$ and $\left(\mathfrak{W}^{\prime}=\left(X^{\prime}, \lambda^{\prime}, \phi^{\prime}\right), \Sigma^{\prime}\right)$ be two Weinstein pairs. Let $\Sigma$ and $\Sigma^{\prime}$ be decomposed as $\Sigma=\Sigma_{-} \cup \Sigma_{+}, \Sigma^{\prime}=\Sigma_{-}^{\prime} \cup \Sigma_{+}^{\prime}$ by splitting hypersurfaces $T \subset \Sigma$ and $T^{\prime} \subset \Sigma^{\prime}$, see Section 3.1. Suppose that

- there exists a Weinstein isomorphism $F: \Sigma_{+} \rightarrow \Sigma_{-}^{\prime}$,

$-\Sigma_{-}$is loose in $\partial X$ and

- pairs $\left(\mathfrak{W}, \Sigma_{-}\right)$and $\left(\mathfrak{W}^{\prime}, \Sigma_{+}^{\prime}\right)$ are flexible.

Then the glued pair $(\mathfrak{W}, \Sigma) \underset{F, T, T^{\prime}}{\cup}\left(\mathfrak{W}^{\prime}, \Sigma^{\prime}\right)$ is flexible. In particular, the result of gluing of two flexible Weinstein domains along Weinstein hypersurfaces one of which is loose is flexible. 
This follows from the fact that the gluing operations of two Weinstein pairs can be decomposed into a sequence of handle attachments, and the looseness assumption for the Weinstein hypersurface in one of the glued parts implies that all the critical handles are attached along loose knots.

As a corollary Proposition 4.6 implies the following generalization of the following result of E. Murphy and K. Siegel, [37]:

Proposition 4.7. The product of two Weinstein pairs, one of which is flexible, is flexible.

Indeed, the product of two Weinstein pairs can always be built by a sequence of gluing of various stabilizations of the first pair.

\section{Lagrangian submanifolds of Weinstein domains}

In this section we discuss exact Lagrangian submanifolds in a Weinstein domain $(X, \lambda, \phi)$. The Lagrangians will always be assumed either closed or with Legendrian boundary in $\partial L \subset \partial X$.

Let $\Sigma(\partial L)$ be the Weinstein thickening of the (possibly empty) Legendrian boundary $\partial L$. A Lagrangian $L$ is called regular, see [18], if the Weinstein pair $(X, \Sigma(\partial L))$ admits a skeleton which contains $L$.

Problem 5.1. Are there non-regular exact Lagrangians?

The problem is widely open. While no examples of non-regular Lagrangians are known, in the opposite direction in the case of a closed exact Lagrangian $L$ in a general Weinstein domain $X$ it is even unknown whether $L$ realizes a non-zero homology class in $H_{n}(X)$ (which is a necessary condition for its regularity).

If $L \subset X$ is regular then by removing its tubular neighborhood $N(L)$ one gets a Weinstein cobordism $X_{L}:=\left(W \backslash N(L), \partial_{-} X_{L}:=\overline{\partial N(L) \backslash \partial X}, \partial_{+} X_{L}:=\overline{\partial X \backslash N(L)}\right)$ (between manifolds with boundary if $\partial L \neq \varnothing$ ) whose negative boundary is the unit cotangent bundle of $L$. The Lagrangian $L$ is called flexible, see [18]), if the cobordism $X_{L}$ is flexible.

It was shown in [18] that any flexible $(X, \lambda)$ admits a surprising abundance of flexible Lagrangians with non-empty Legendrian boundary. In particular,

Theorem 5.2. Let $L$ be an n-manifold with non-empty boundary, equipped with a fixed trivialization $\eta$ of its complexified tangent bundle $T L \otimes \mathbb{C}$. Then there exists 
a flexible Lagrangian embedding with Legendrian boundary $(L, \partial L) \rightarrow\left(B^{2 n}, \partial B^{2 n}\right)$ where $B^{2 n}$ is the standard symplectic $2 n$-ball, realizing the trivialization $\eta$. In particular, any 3-manifold with boundary can be realized as a flexible Lagrangian submanifold of $B^{6}$ with Legendrian boundary in $\partial B^{6}$.

\section{Symplectic topology of Weinstein manifolds}

While flexible Weinstein structures enjoy a full parametric $h$-principle, there is plenty of symplectic rigidity and fine symplectic invariants of non-flexible ones. I will not discuss in this survey any such invariants and just mention that until recently most examples of formally homotopic but not symplectomorphic Weinstein manifolds were distinguished by their (possibly appropriately deformed) symplectic cohomology. For instance, there are infinitely many non-symplectomorphic Weinstein structures on $\mathbb{R}^{2 n}$ for any $n>2([41,33)$ and by taking connected sums of these examples with flexible Weinstein manifolds one gets infinitely many non-symplectomorphic Weinstein structures on any given "almost Weinstein" (i.e. an almost complex manifold of homotopy type of a half-dimensional CW-complex) manifolds, see [2] .

Note that Theorem 5.2 can also be used for constructing exotic Weinstein structures. In particular,

Theorem 6.1 ([18]). Let $L$ be a closed 3-manifold. Then there exists a unique up so symplectomorphism Weinstein structure $\mathfrak{W}(L)=\left(\omega_{L}, Z_{L}, \phi_{L}\right)$ on $T^{*} S^{3}$ which contains $L$ as its flexible Lagrangian submanifold in the homology class of the 0section (with $\mathbb{Z} / 2$-coefficients in the non-orientable case). Moreover, infinitely many of these $\mathfrak{W}(L)$ are pairwise non-symplectomorphic.

Note that there exists only 1 homotopy class of almost complex structures on $T^{*} S^{3}$. While the symplectic structure of $\mathfrak{W}(L)$ carries a lot of information about the topology of $L$, the following problem is open:

Problem 6.2. Suppose $\mathfrak{W}(L)$ is symplectomorphic to $\mathfrak{W}\left(L^{\prime}\right)$ ? Does it imply that $L$ is diffeomorphic to $L^{\prime}$ ?

The famous "nearby Lagrangian problem" asks whether there is a unique up to Hamiltonian isotopy exact closed Lagrangian submanifold in the standard $T^{*} M$ for a closed $M$. Though in this form the answer is unknown except for $M=S^{2}$ and $T^{2}$, see [27, 12, the answer is positive up to simple homotopy equivalence, [1], and hence according to Smale, Freedman and Perelman for $M=S^{n}$ up to homeomorphism, 
and for some dimensions, e.g. $n=3,5,6,12$, even up to diffeomorphism, [34]. As it was pointed out to me by O. Lazarev, one can show using methods of [9] that certain exotic $T^{*} S^{n}$ may contain several not homotopy equivalent regular closed exact Lagrangian submanifolds.

Problem 6.3. Can the uniquenes results from [1] be extended to a more general class of Weinstein structures on $T^{*} S^{n}$ ?

The proof of Theorem 5.2 yields also the following slightly stronger result.

Theorem 6.4. Let $(X, \omega, \lambda, \phi)$ be a 6-dimensional Weinstein domain such that $\phi$ has exactly 1 critical point of index 3 (and any number of critical points of smaller indices). Suppose also that the symplectic vector bundle $(T X, d \lambda)$ is trivial. Then there exists a Weinstein structure $\left(\omega_{X}, \lambda_{X}, \phi_{X}\right)$ on $T^{*} S^{3}$ which admits an embedding

$$
(X, \omega, \lambda, \phi) \rightarrow\left(T^{*} S^{3}, \omega_{X}, \lambda_{X}, \phi_{X}\right)
$$

onto a Weinstein subdomain with a flexible complement.

\section{Topology of Weinstein fillings}

Contact manifolds appeared as boundaries of Weinstein domains are called Weinstein fillable. The fact that a Weinstein filling has a homotopy type of a half-dimensional CW-complex imposes constraints on the topology of its contact boundary and the stable almost complex class which can be realized by Weinstein fillable contact structures on a given smooth manifold. This question was studied in detail by BowdenCrowley-Stipsicz in [5, 6]. In particular, they showed that there are classes of homotopy spheres which do not admit any Weinstein fillable contact structure.

Given a contact manifold $(Y, \xi)$ one can try to describe (symplectic) topology of its Weinstein fillings. In this section we discuss this problem for contact manifolds of dimension $2 n-1>3$, see [40] for a survey of results for 3-dimensional manifolds.

First of all notice that the fact that $X$ retracts to its $n$-dimensional skeleton implies that the inclusion $Y=\partial X \hookrightarrow X$ is $(n-1)$-connected, and in particular, if $Y$ is a homotopy sphere then $X$ is $(n-1)$-connected. It turns out that some contact structures know much more about the topology of their fillings.

Theorem 7.1 ([31]). Any Weinstein filling of the standard contact sphere $\left(S^{2 n-1}, \xi_{\mathrm{std}}\right)$ is diffeomorphic to the ball $B^{2 n}$. 
Generalizing Theorem 7.1 K. Barth, H. Geiges and K. Zehmisch proved in [7]:

Theorem 7.2. All Weinstein fillings of a simply connected contact manifold admitting a subcritical filling are diffeomorphic.

In fact, both Theorems 7.1 and 7.2 hold in a stronger form for a more general class of symplectic, and not necessarily Weinstein fillings. We also note that while it follows from Theorem 4.1 that all completed subcritical Weinstein fillings of a given contact manifold are symplectomorphic (we note that the $(n-1)$-connectedness of the inclusion map $\partial X \hookrightarrow X$ implies that the homotopy class of an almost complex structure on a subcritical manifold is determined by the homotopy class of its restriction to the boundary), it is unknown for $n>2$ whether all completed fillings of a contact manifold admitting a subcritical filling (e.g. the standard contact sphere) are symplectomorphic.

The following theorem of Oleg Lazarev constrains topology of flexible Weinstein manifolds.

Theorem 7.3 ([29]). All flexible fillings of of a contact manifold $(Y, \xi)$ with $c_{1}(Y, \xi)=$ 0 have canonically isomorphic integral homology.

In particular, as Lazarev observed, Theorem 7.3 together Smale's classification of 2-connected 6-manifolds from [43] and the fact that $\pi_{3}(O / U)=0$ yield a complete classification of flexibly fillable contact structures on $S^{5}$.

Corollary 7.4 ([29]). There exists a sequence $\xi_{n}, n=0,1, \ldots$, of pairwise noncontactomorphic contact structures on $S^{5}$ such that

- any flexibly fillable contact structure on $S^{5}$ is contactomorphic to one of the structures from this sequence;

- the contact structure $\xi_{0}$ is standard;

- for $n \geq 1$ the contact sphere $\left(S^{5}, \xi_{n}\right)$ admits a unique up to symplectomorphism flexible Weinstein filling diffeomorphic to $\left.\underset{1}{\#} S^{3} \times S^{3}\right) \backslash B^{6}$.

There are further constraints on the topology of flexible Weinstein fillings. In particular,

Theorem 7.5 ([19]). Let $\left(S^{4 n-1}, \xi\right)$ be a flexibly fillable contact structure. Then the signature of its flexible filling is uniquely determined by the contact structure $\xi$. 
Problem 7.6. Does a contact structure $(Y, \xi)$ remember

a) the diffeomorphism type of its flexible Weinstein filling $(X, \omega, Z, \phi)$ ?

b) the almost symplectic homotopy class $[\omega]$ of the symplectic structure $\omega$ ?

We note that the diffeomorphism type of $X$ together with the homotopy class $[\omega]$ determine a flexible Weinstein structure up to Weinstein homotopy, and hence the positive answer to a) and b) would imply that the contact structure $(Y, \xi)$ remember the symplectomorphism type of the completion of its flexible filling.

\section{Nadler's program of arborealization}

A priori, a skeleton of a Weinstein domain can have very complicated singularities. However, David Nadler conjectured that up to Weinstein homotopy the singularities of the skeleton can be reduced to a finite list in any dimension, see 38. For $2 n$ dimensional symplectic Weinstein manifolds the list of Nadler's singularities, which he calls arboreal, are enumerated by decorated rooted trees with $\leq n+1$ vertices. It is remarkable that the singularity of each given type has a unique symplectic realization. Nadler also proposed in [39] a procedure for arborealization of the skeleton of a Weinstein structure. His procedure replaces a given Weinstein structure by another one whih an arboreal skeleton. Nadler proved in [39] that the constructed Weinstein manifold has microlocal sheaf-theoretic invariants equivalent to those of the Weinstein manifold. Conjecturally this implies that the wrapped Fukaya categories are also the same for the original and modified Weinstein manifold. However, it is unclear whether Nadler's modification yields a Weinstein structure which is homotopic, or even symplectomorphic to the original one.

In an ongoing joint project [23] with David Nadler and Laura Starkston we are exploring a somewhat different strategy for arborealization of the Weinstein skeleton via a Weinstein homotopy using simplification of singularities type technique in the spirit of a recent paper of D. Álvarez-Gavela, [4]. In some special cases this program was already carried out by Starkston in [44].

In this section we discuss the arboreal singularities with more detail and give precise statements of some of the results from [23]. 


\subsection{Definition of an arboreal singularity}

While we define below arboreal models as closed properly embedded subsets of the standard symplectic vector space, we are interested only in germs of these models at the origin.

Consider a tree $T$ with $\leq n+1$ vertices and a fixed vertex $R$, the root. Suppose in addition that all edges, except the terminal ones are decorated with \pm 1 . We will denote by $\varepsilon$ the decoration, and by $|T|$ the total number of vertices. With each decorated rooted tree $(T, \varepsilon)$ we associate a unique up to symplectomorphism model $A(T, \varepsilon, m) \subset \mathbb{R}^{2 m}=T^{*} \mathbb{R}^{m}$ in each dimension $m \geq n$ of the skeleton. The models will be stratified by strata which are isotropic for the Liouville form $p d q$. In dimension $m>n$ we have $A(T, \varepsilon, m)=A(T, \varepsilon, n) \times \mathbb{R}^{m-n} \subset T^{*} \mathbb{R}^{n} \times T^{*} \mathbb{R}^{m-n}=T^{*} \mathbb{R}^{m}$.

The model $A(T, \varepsilon, n)$ will be defined inductively in $n$. For a tree $T$ which consists of one vertex we define $A(T, 0)$ to be a point (in the 0 -dimensional symplectic space $\left.T^{*} \mathbb{R}^{0}\right)$, and respectively $A(T, n)=\mathbb{R}^{n} \subset T^{*} \mathbb{R}^{n}$.

As it was already stated above, the Liouville form $p d q$ vanishes on each stratum of the model $A(T, \varepsilon, n) \subset T^{*} \mathbb{R}^{n}$. Hence, if we view $T^{*} \mathbb{R}^{2 n}$ as a (Weinstein) hypersurface $\{z=0\}$ in the contact space $\left(\mathbb{R}^{2 n+1}=T^{*} \mathbb{R}^{n} \times \mathbb{R}, p d q+d z\right)$, then all strata of $A(T, \varepsilon, n) \subset T^{*} \mathbb{R}^{n}$ are also isotropic for the contact form $p d q+d z$. However, unless $A(T, \varepsilon, n)$ is a Lagrangian plane, the front projection $(p, q, z) \mapsto(q, z)$ is very degenerate, because it collapses the image to the hyperplane $\{z=0\}$. We want to deform the model $A(T, \varepsilon, n)$ in $\mathbb{R}^{2 n+1}$ to make the front projection more generic. To do that, consider a contactomorphism $S: \mathbb{R}^{2 n+1} \rightarrow \mathbb{R}^{2 n+1}$ given by the formula

$$
S\left(p_{1}, \ldots, p_{n}, q_{1}, q_{2}, \ldots, q_{n}, z\right)=\left(p_{1}, \ldots, p_{n}, q_{1}+p_{1}, q_{2}, \ldots, q_{n}, z-\frac{p_{1}^{2}}{2}\right) .
$$

Then

$$
S^{-1}\left(p_{1}, \ldots, p_{n}, q_{1}, q_{2}, \ldots, q_{n}, z\right)=\left(p_{1}, \ldots, p_{n}, q_{1}-p_{1}, q_{2}, \ldots, q_{n}, z+\frac{p_{1}^{2}}{2}\right) .
$$

Denote

$$
\widehat{A}^{+}(T, \varepsilon, n):=S(A(T, \varepsilon, n)), \quad \widehat{A}^{-}(T, \varepsilon, n):=S^{-1}(A(T, \varepsilon, n)) .
$$

The sets $\widehat{A}^{ \pm}(T, \varepsilon, n)$ are stratified by isotropic for the contact form $d z+p d q$ strata. If $|T|=1$ we have $\widehat{A}^{+}(T, n)=A(T, n)$.

Suppose that we already defined models for all decorated rooted trees $(T, \varepsilon)$ with $|T| \leq n$. Consider a rooted tree $(T, \varepsilon)$ with $|T|=n+1$. By removing the root $R$ and all edges adjacent to $R$ we get $k$ decorated trees $\left(T_{1}, \varepsilon\right), \ldots,\left(T_{k}, \varepsilon_{k}\right)$ with 

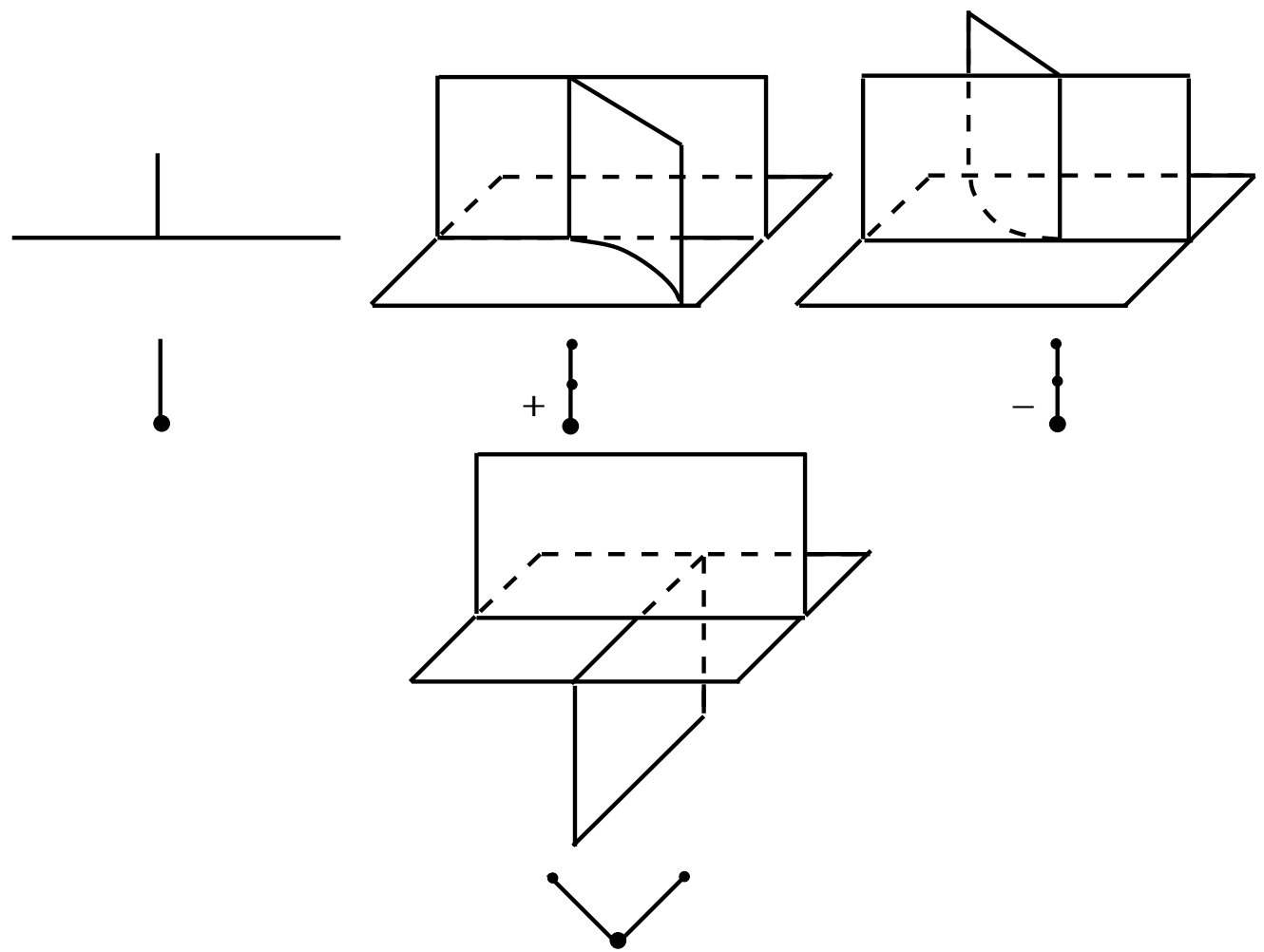

Fig. 8.1: Arboreal singularities labeled by rooted decorated trees. The picture represents Lagrangian skeleta themselves, and not their front projections. Free boundaries of vertical strata form Legendrian trees, while their traces at the horizontal plane are fronts of these trees.

$\left|T_{1}\right|=n_{1}, \ldots,\left|T_{k}\right|=n_{k}, n_{1}+\cdots+n_{k}=n$. For each of them we choose as its root the vertex which was connected in $T$ to $R$. Let $\sigma_{j}= \pm 1$ be the decoration of the edge which was connecting the root $R$ with the root of the tree $T_{j}, j=1, \ldots, k$.

Consider already defined models $A\left(T_{1}, \varepsilon_{1}, n-1\right), \ldots, A\left(T_{k}, \varepsilon_{k}, n-1\right) \subset T^{*} \mathbb{R}^{n-1} \times \mathbb{R}$.

Denote $N_{0}:=0, N_{j}:=\sum_{i=1}^{j} n_{i}, j=1, \ldots, k-1$. For each $j=0, \ldots, k-1$ consider the hyperplane $\Pi_{j}=\left\{p_{N_{j}+1}=1\right\}$ in $\mathbb{R}^{2 n}=T^{*} \mathbb{R}^{n}$ with the Liouville form $\lambda=\sum_{1}^{n} p_{j} d q_{j}$. Note that $\Pi_{j}$ is transverse to the Liouville vector field $Z=\sum_{1}^{n} p_{j} \frac{\partial}{\partial p_{j}}$, or equivalently 
$\left.\lambda\right|_{\Pi_{j}}=d q_{N_{j}+1}+\sum_{i \in\{1, \ldots, n\}, i \neq N_{j}+1} p_{i} d q_{i}$ is a contact form. Cyclically ordering coordinates $q_{N_{j}+2}, \ldots, q_{n}, q_{1}, \ldots, q_{N_{j}}$ and taking the coordinate $q_{N_{j}+1}$ as $z$ we identify $\Pi_{j}$ with $T^{*} \mathbb{R}^{n-1} \times \mathbb{R}$. Consider $A^{\operatorname{sign}\left(\sigma_{j}\right)}\left(T_{j}, \varepsilon_{j}, n-1\right) \subset \Pi_{j}$.

Denote

$$
\left.B(T, \varepsilon, n):=\left\{(t p, q) \in T^{*} \mathbb{R}^{n} ; t \in[0, \infty),(p, q) \in \bigcup_{j=1}^{k} \widehat{A}^{\operatorname{sign}\left(\sigma_{j}\right)}\left(T_{j}, \varepsilon_{j}, n-1\right)\right)\right\} .
$$

Note that $B(T, \varepsilon, n) \cap\{p=0\}$ is the union of front projections of Legendrian complexes $\left.\widehat{A}^{\text {sign }\left(\sigma_{j}\right)}\left(T_{j}, \varepsilon_{j}, n-1\right)\right)$, and $B(T, \varepsilon, n)$ is the positive conormal of this stratified set co-oriented by the vector field $\frac{\partial}{\partial q_{N_{j}+1}}$. Finally, we define

$$
A(T, \varepsilon, n):=\{p=0\} \cup B(T, \varepsilon, n) .
$$

Singularities of the form $A(T, \varepsilon, n)$ where $(T, \varepsilon)$ is a decorated rooted tree are called primary arboreal.

Note that up to linear symplectomorphism the result of the above construction is independent of the ordering of the trees $T_{1}, \ldots, T_{k}$. Indeed, the corresponding symplectomorphism is the symplectization of the linear automorphism of $\mathbb{R}^{n}$ appropriately permuting the coordinates $q_{1}, \ldots, q_{n}$.

As an example, let us explicitly construct the models shown on Fig, 8.1. For a tree with 2 vertices we take the standard symplectic $\mathbb{R}^{2}$ with coordinates $(p, q)$. Then $\Pi=\{p=1\}$. For the 1 -vertex tree $T_{1}$ the model $A\left(T_{1}, 0\right)$ coincides is the point $\{p=1, q=0\} \in \Pi$ and $\widehat{A}\left(T_{1}, 0\right)=A\left(T_{1}, 0\right)$. Hence $B(T, 1)=\{(t, 0), t \geq 0\}$ is the positive $p$-semi-axis, and $A(T, 1)=\{p=0\} \cup B(T, 2)$, is the union of the coordinate line $q$ with this semi-axis, as it is shown on the left side of Fig. 8.1.

For the rooted tree with three vertices and the central root, as on the lower picture in Fig. 8.1, each of the trees $T_{1}, T_{2}$ has 1 vertex. Hence, $\Pi_{1}=\left\{p_{1}=1\right\}, \Pi_{2}=\left\{p_{2}=1\right\}$, and identifying this hyperplanes with the standard contact $\mathbb{R}^{3}$ we get $A\left(T_{1}, 1\right)=$ $\left\{p_{2}=q_{1}=0\right\} \subset \Pi_{1}$ and $A\left(T_{2}, 1\right)=\left\{p_{1}=q_{2}=0\right\} \subset \Pi_{2}$. Therefore,

$$
A(T, 2)=\{p=0\} \cup\left\{p_{2}=q_{1}=0, p_{1} \geq 0\right\} \cup\left\{p_{1}=q_{2}=0, p_{2} \geq 0\right\} .
$$

Finally, consider the right models on Fig. 8.1. The models are contained in the standard symplectic $\mathbb{R}^{4}$ with canonical coordinates $\left(p_{1}, q_{1}, p_{2}, q_{2}\right)$, and we have $\Pi=$ $\Pi_{1}=\left\{p_{1}=1\right\}$ The tree $T_{1}$ in this case consists of two vertices, and identifying $\Pi$ with the standard symplectic $\mathbb{R}^{2}$, we find that

$$
\widehat{A}^{ \pm}\left(T_{1}, 1\right)=\left\{q_{1}=p_{2}=0\right\} \cup\left\{q_{1}=\mp p_{2}^{2}, p_{2}= \pm q_{2}, p_{2} \geq 0\right\} .
$$


Note that the second stratum in the union can also be written as $\left\{q_{1}=\mp q_{2}^{2}, p_{2}=\right.$ $\left.\pm q_{2}, p_{2} \geq 0\right\}$ Thus we have

$$
\begin{aligned}
& B(T,+1,2)=\left\{p_{2}=0, q_{1}=0, p_{1} \geq 0\right\} \cup\left\{q_{1}=-\frac{q_{2}^{2}}{2}, p_{2}=p_{1} q_{2}, p_{1}, p_{2} \geq 0\right\} \\
& B(T,-1,2)=\left\{p_{2}=0, q_{1}=0, p_{1} \geq 0\right\} \cup\left\{q_{1}=\frac{q_{2}^{2}}{2}, p_{2}=-p_{1} q_{2}, p_{1}, p_{2} \geq 0\right\}
\end{aligned}
$$

Note that $B(T, \pm 1,2) \cap\{p=0\}=\left\{q_{2}=0\right\} \cup\left\{q_{1}=\mp \frac{q_{2}^{2}}{2}\right\}$ is the front of the Legendrian tree $\widehat{A}^{ \pm}\left(T_{1}, 1\right)$, while $B(T, \pm 1,2)$ is the positive conormal of this front co-oriented by the vector field $\frac{\partial}{\partial q_{1}}$.

A general arboreal singularity is associated to a double decorated rooted tree with an additional decoration $\beta$ which assigns 0 or 1 to all terminal vertices of the tree $T$. We extend $\beta$ to all vertices by setting $\beta(v)=0$ for all non-terminal vertices. Primary arboreal singularities correspond to the case when the decoration $\beta$ is identically 0 .

We denote $|\beta|:=\sum \beta(v)$, where the sum is taken over all terminal vertices $v$ of the tree $T$. With each double decorated tree $(T, \varepsilon, \beta)$ we associate a unique up to symplectomorphism model $A(T, \varepsilon, \beta, m) \subset T^{*} \mathbb{R}^{m}$ for each $m \geq|T|+|\beta|-1$. In dimension $m \geq n:=|T|+|\beta|-1$ we have

$$
A(T, \varepsilon, \beta, m)=A(T, \varepsilon, \beta, n) \times \mathbb{R}^{m-n} \subset T^{*} \mathbb{R}^{n} \times T^{*} \mathbb{R}^{m-n}=T^{*} \mathbb{R}^{m} .
$$

The model $A(T, \varepsilon, \beta, m) \subset T^{*} \mathbb{R}^{m}$ with $m=|T|+|\beta|-1$ is defined by a similar inductive procedure as for primary arboreal singularities, beginning with

$$
A(T, \varepsilon, \beta, 1)=\{p=0, q \geq 0\} \subset T^{*} \mathbb{R} \text { for }|T|=1 \text { and }|\beta|=1 .
$$

Every model $A(T, \varepsilon, \beta, m) \subset T^{*} \mathbb{R}^{m}$ can be presented as a union of Lagrangian sheets $L_{v}$ enumerated by vertices of the graph $T$. Denote by $d(v)$ the distance between $v$ and the root. Then $L_{v}$ is diffeomorphic to the quadrant

$$
\left\{\left(x_{1}, \ldots, x_{n}\right) \in \mathbb{R}^{n} ; x_{1}, \ldots x_{k} \geq 0\right\}, \quad k=d(v)+\beta(v) .
$$

Note that the model $A(T, \varepsilon, \beta, m)$ inherits a smooth structure (i.e. the algebra of smooth functions) from the ambient space $\mathbb{R}^{2 n}$. By an $n$-dimensional arboreal complex we mean a set covered by charts diffeomorphic to one of the models $A(T, \varepsilon, \beta, n)$. Hence, every arboreal complex can be canonically stratified by strata $S_{T, \varepsilon, \beta}$ of dimension $n-|T|-|\beta|+1$. A diffeomorphism $f: C \rightarrow C^{\prime}$ between two arboreal complexes induces a diffeomorphism between the corresponding strata, but not every continuous map $f: C \rightarrow C^{\prime}$ which is a diffeomorphism on the corresponding strata is a diffeomorphism of arboreal complexes $C$ and $C^{\prime}$. 


\subsection{Main results}

Proposition 8.1 ([23]). For each arboreal complex $C$ there exists a unique up to symplectomorphism Weinstein domain $\mathfrak{W}(C)=(X, \omega, Z, \phi)$, "the cotangent bundle" of $C$ such that $C=\operatorname{Core}(\mathrm{X}, \omega, \mathrm{Z})$. Any two such Weinstein structures $(X, \omega, Z, \phi)$ and $\left(X, \omega, Z^{\prime}, \phi^{\prime}\right)$ are homotopic through a family of Weinstein structures with a fixed core.

Theorem $8.2([23])$. (i) Any Weinstein structure is homotopic to a Weinstein structure with an arboreal skeleton.

(ii) Let $\mathfrak{W}_{t}, t \in[0,1]$ be a Weinstein homotopy such $\mathfrak{W}_{0}$ and $\mathfrak{W}_{1}$ have arboreal skeleta. Then there exists a Weinstein pair structure $\left(\mathfrak{W} ; \mathfrak{W}_{0} \cup \mathfrak{W}_{1}\right)$ on $X \times T^{*} I$ with an arboreal skeleton which is homotopic to the Weinstein pair associated to the homotopy $\mathfrak{W}_{t}$ (see Section 3.3. .

Under some topological constraints on the manifold $X$ one can further restrict the list of necessary singularities.

Theorem $8.3([23])$. Let $\mathfrak{W}=(X, \omega, Z, \phi)$ be a Weinstein structure. Suppose that

a) the manifold $X$ is $(n-2)$-connected;

b) there exists a field of Lagrangian planes $\tau \subset T X$; in other words, $T X$ with its homotopically canonical almost complex structure is isomorphic to the complexification of a real $n$-dimensional vector bundle.

Then the Weinstein structure $\mathfrak{W}$ is homotopic to a Weinstein structure $\widetilde{\mathfrak{W}}=(X, \omega, \widetilde{Z}, \widetilde{\phi})$ whose skeleton is an arboreal complex with singularities of type $(T, \varepsilon, \beta)$ where the distance from the root of the tree $T$ to any other vertex is no more than 2 and the decoration $\varepsilon$ takes only positive values.

\section{References}

[1] M. Abouzaid and T. Kragh, Simple homotopy equivalence of nearby Lagrangians, arXiv:1603.05431.

[2] M. Abouzaid and P. Seidel, Altering symplectic manifolds by homologous recombination, arXiv:1007.3281. 
[3] R. Avdek, Liouville hypersurfaces and connect sum cobordisms, arXiv:1204.3145.

[4] D. Álvarez-Gavela, The simplification of singularities of Lagrangian and Legendrian fronts, arXiv:1605.07259.

[5] J. Bowden, D. Crowley and A. Stipsicz, The topology of Stein fillable manifolds in high dimensions I, arXiv:arXiv:1306.2746.

[6] J. Bowden, D. Crowley, A. Stipsicz, The topology of Stein fillable manifolds in high dimensions II, Geom. Topol. 19(2015), 2995-3030.

[7] K. Barth, H. Geiges and K. Zehmisch, The diffeomorphism type of symplectic fillings, arXiv:1607.03310.

[8] F. Bourgeois, T. Ekholm and Y. Eliashberg, Effect of Legendrian Surgery, Geom. Topol., 16(2012), 301-389.

[9] C. Cao, N. Gallup, K. Hayden, J. Sabloff, Topologically Distinct Lagrangian and Symplectic Fillings, arXiv:1307.7998.

[10] K. Cieliebak, Handle attaching in symplectic homology and the Chord Conjecture, J. Eur. Math. Soc.. 4(2002), 115-142.

[11] K. Cieliebak and Y. Eliashberg, From Stein to Weinstein and Back - Symplectic Geometry of Affine Complex Manifolds, Colloquium Publications Vol. 59, Amer. Math. Soc. (2012).

[12] G. Dimitroglou Rizell, E. Goodman and A. Ivrii, Lagrangian isotopy of tori in $S^{2} \times S^{2}$ and $\mathbb{C} P^{2}$, arXiv:1602.08821.

[13] T. Ekholm, Rational SFT, linearized Legendrian contact homology, and Lagrangian Floer cohomology, Perspectives in analysis, geometry, and topology, 109-145, Progr. Math., 296, Birkhäuser/Springer, New York, 2012.

[14] T. Ekholm and Y. Lekili, Duality between Lagrangian and Legendrian invariants, arXiv:1701.01284.

[15] Y. Eliashberg, Symplectic geometry of plurisubharmonic functions, in "Gauge Theory and Symplectic Geometry", Vol. 488 of the series NATO ASI Series, 49-67.

[16] Y. Eliashberg, Topological characterization of Stein manifolds of dimension $>2$, Internat. J. Math. 1(1990), no. 1, 29-46. 
[17] Y. Eliashberg, Classification of contact structures on $\mathbb{R}^{3}$, Int. Math. Res. Notices, 1993, no. 3, 87-91.

[18] Y. Eliashberg, S. Ganatra and O. Lazarev, Flexible Lagrangians, arXiv:1510.01287.

[19] Y. Eliashberg, S. Ganatra and O. Lazarev, Topology of flexible fillings, in preparation.

[20] Y. Eliashberg, M Gromov, Convex symplectic manifolds, Proc. Symp. Pure Math., 52(1991), Amer. Math. Soc., Providence, RI, 135-162.

[21] Y. Eliashberg, M Gromov, Lagrangian intersection theory. Finite-dimensional approach, AMS Transl., 186(1998), N2, 27-116.

[22] Y. Eliashberg and E. Murphy, Lagrangian caps, Geom. and Funct. Anal., 23(2013), Volume 23, 1483-1514.

[23] Y. Eliashberg, D. Nadler and L. Starkston, in preparation.

[24] E. Giroux, Convexité en topologie de contact, Comment. Math. Helv., 66 (1991), $637-677$.

[25] E. Giroux, Géométrie de contact: de la dimension trois vers les dimesions supérieures, Proc. of the ICM, Vol. II (Beijing, 2002), Higher Ed. Press, Beijing, 2002, 405-414.

[26] S. Ganatra, J. Pardon and V. Shende, Covariantly functorial Floer theory on Liouville sectors, arXiv:1706.03152.

[27] R. Hind, Lagrangian spheres in $S^{2} \times S^{2}$, Geom. Funct. Anal., 14(2004), 303-318.

[28] M. Kontsevich, Symplectic geometry of homological algebra, http://www.ihes.fr/ maxim/TEXTS/Symplectic_AT2009.pdf.

[29] O. Lazarev, Contact manifolds with flexible fillings, arXiv:1610.04837.

[30] F. Laudenbach, On the Thom-Smale complex, an Appendix to Bismut-Zhang, An extension of a Theorem by Cheeger and Müller, Astérisque 205(1992).

[31] D. McDuff, Symplectic manifolds with contact type boundaries, Invent. Math. 103(1991), 651-671. 
[32] M. Maydanskiy and P. Seidel, Lefschetz fibrations and exotic symplectic structures on cotangent bundles of spheres, J. Topol. 3 (2010), 157-180.

[33] M. McLean, Lefschetz fibrations and symplectic homology, Geom. Topol. 13(2009), 1877-1944.

[34] M. Kervaire, and J.W. Milnor, Groups of homotopy spheres: I. Ann. of Math., $77(3), 504-537$.

[35] E. Murphy, Loose Legendrian embeddings in high dimensional contact manifolds, arXiv:1201.2245.

[36] E. Murphy, Closed exact Lagrangians in the symplectization of contact manifolds, arXiv:1304.6620.

[37] E. Murphy and K. Siegel, Subflexible symplectic manifolds, arXiv:1510.01867.

[38] D. Nadler, Arboreal Singularities, arXiv:1309.4122

[39] D. Nadler, Non-characteristic expansion of Legendrian singularities, arXiv:1507.01513.

[40] B. Ozbagci, On the topology of fillings of contact 3-manifolds, http://home.ku.edu.tr/ bozbagci/SurveyFillings.pdf.

[41] P. Seidel and I. Smith, The symplectic topology of Ramanujams surface, Comment. Math. Helv. 80(2005), 859-881.

[42] Z. Sylvan, On partially wrapped Fukaya categories, arXiv: 1604:02540v2.

[43] S. Smale, On the structure of manifolds, Amer. J. Math., 84(1962) pp. 387-399.

[44] L. Starkston, Arboreal Singularities in Weinstein Skeleta, arXiv:1707.03446.

[45] A. Weinstein, Contact surgery and symplectic handlebodies, Hokkaido Math. J. 20(1991), 241-251. 